\title{
RELATIONS BETWEEN ABS-NORMAL NLPS AND MPCCS. PART 2: WEAK CONSTRAINT QUALIFICATIONS
}

\author{
L.C. Hegerhorst-Schultchen ${ }^{*} \quad$ C. Kirches $^{\dagger} \quad$ M.C. Steinbach ${ }^{\dagger}$
}

\begin{abstract}
This work continues an ongoing effort to compare non-smooth optimization problems in abs-normal form to Mathematical Programs with Complementarity Constraints (MPCCs). We study general Nonlinear Programs with equality and inequality constraints in abs-normal form, so-called Abs-Normal NLPs, and their relation to equivalent MPCC reformulations. We introduce the concepts of Abadie's and Guignard's kink qualification and prove relations to MPCC-ACQ and MPCC-GCQ for the counterpart MPCC formulations. Due to non-uniqueness of a specific slack reformulation suggested in [10], the relations are non-trivial. It turns out that constraint qualifications of Abadie type are preserved. We also prove the weaker result that equivalence of Guginard's (and Abadie's) constraint qualifications for all branch problems hold, while the question of GCQ preservation remains open. Finally, we introduce M-stationarity and B-stationarity concepts for abs-normal NLPs and prove first order optimality conditions corresponding to MPCC counterpart formulations.
\end{abstract}

\section{INTRODUCTION}

Non-smooth nonlinear optimization problems of the form

$$
\min _{x} f(x) \quad \text { s.t. } \quad g(x)=0, \quad h(x) \geq 0,
$$

where $D^{x} \subseteq \mathbb{R}^{n}$ is open, the objective $f \in C^{d}\left(D^{x}, \mathbb{R}\right)$ is a smooth function and the equality and inequality constraints $g \in C_{\text {abs }}^{d}\left(D^{x}, \mathbb{R}^{m_{1}}\right)$ and $h \in C_{\text {abs }}^{d}\left(D^{x}, \mathbb{R}^{m_{2}}\right)$ are level-1 non-smooth functions that can be written in abs-normal form [3] have been considered by the authors in [10]. In this problem class, the non-smoothness is caused by finitely many occurrences of the absolute value function, the branches of which we represent by signature matrices $\Sigma=\operatorname{diag}(\sigma)$ with $\sigma \in\{-1,0,1\}^{s}$. We find functions $c_{\mathcal{E}} \in C^{d}\left(D^{x,|z|}, \mathbb{R}^{m_{1}}\right), c_{I} \in C^{d}\left(D^{x,|z|}, \mathbb{R}^{m_{2}}\right)$ and $c_{\mathcal{Z}} \in C^{d}\left(D^{x,|z|}, \mathbb{R}^{s}\right)$ with $D^{x,|z|}=D^{x} \times D^{|z|}$ $D^{|z|} \subseteq \mathbb{R}^{s}$ open and symmetric (i.e., $z \in D^{|z|}$ implies $\Sigma z \in D^{|z|}$ for every signature matrix $\Sigma$ ) such that

$$
\begin{aligned}
g(x) & =c_{\mathcal{E}}(x,|z|), \\
h(x) & =c_{\mathcal{I}}(x,|z|), \\
z & =c_{\mathcal{Z}}(x,|z|) \quad \text { with } \partial_{2} c_{\mathcal{Z}}(x,|z|) \text { strictly lower triangular. }
\end{aligned}
$$

C. Kirches discloses support by the German Federal Ministry of Education and Research through grants ${ }^{\circ}{ }_{0} \mathrm{M}_{17} \mathrm{MBA}^{-}$ MoPhaPro, o5M18MBA-MOReNet, and o1/S17089C-ODINE, and by Deutsche Forschungsgemeinschaft (DFG) through Priority Programme 1962, grant Ki1839/1-2.

*Leibniz Universität Hannover, Institut für Angewandte Mathematik, Welfengarten 1, 30167 Hannover, Germany. hegerhorst@ifam.uni-hannover.de

$\dagger$ Technische Universität Carolo-Wilhelmina zu Braunschweig, Institut für Mathematische Optimierung, Universitätsplatz 2, 38106 Braunschweig, Germany. c.kirches@tu-bs.de

¥Leibniz Universität Hannover, Institut für Angewandte Mathematik, Welfengarten 1, 30167 Hannover, Germany. mcs@ifam.uni-hannover.de 
Here we use a single joint switching constraint $c_{\mathcal{Z}}$ for both $g$ and $h$, and reuse switching variables $z_{i}$ if the same argument repeats as an absolute value argument in $g$ or $h$. Due to the strictly lower triangular form of $\partial_{2} c_{\mathcal{Z}}(x,|z|)$, component $z_{j}$ of $z$ can be computed from $x$ and the components $z_{i}, i<j$. Hence, the variable $z$ is implicitly defined by $z=c_{\mathcal{Z}}(x,|z|)$, and to denote this dependence explicitly, we write $z(x)$ in the following. Whenever we address questions of solvability of this system, we make use of the reformulation $\left|z_{i}\right|=\operatorname{sign}\left(z_{i}\right) z_{i}$.

Definition 1.1 (Signature of $z$ ). Let $x \in D^{x}$. We define the signature $\sigma(x)$ and the associated signature matrix $\Sigma(x)$ as

$$
\sigma(x):=\operatorname{sign}(z(x)) \in\{-1,0,1\}^{s} \quad \text { and } \quad \Sigma(x):=\operatorname{diag}(\sigma(x)) .
$$

A signature vector $\sigma(x) \in\{-1,1\}^{s}$ is called definite, otherwise indefinite.

For signatures $\sigma, \hat{\sigma} \in\{-1,0,1\}^{s}$, it is convenient to use the partial order

$$
\hat{\sigma} \geq \sigma \Longleftrightarrow \hat{\sigma}_{i} \sigma_{i} \geq \sigma_{i}^{2} \text { for } i=1, \ldots, s,
$$

i.e., $\hat{\sigma}_{i}$ is arbitrary if $\sigma_{i}=0$ and $\hat{\sigma}_{i}=\sigma_{i}$ otherwise. Thus, we may write $|z(x)|=\Sigma z(x)$ for every $\sigma \geq \sigma(x)$. Further, we may consider the system $z=c_{\mathcal{Z}}(x, \Sigma z)$ for fixed signature $\Sigma=\Sigma(\hat{x})$ around a point of interest $\hat{x}$. By the implicit function theorem, the system has a locally unique solution $z(x)$ for fixed signature $\Sigma$, and the associated Jacobian at $\hat{x}$ reads

$$
\partial_{x} z(\hat{x})=\left[I-\partial_{2} c_{\mathcal{Z}}(\hat{x},|z(\hat{x})|) \Sigma\right]^{-1} \partial_{1} c_{\mathcal{Z}}(\hat{x},|z(\hat{x})|) \in \mathbb{R}^{s \times n} .
$$

Definition 1.2 (Active Switching Set). We call the switching variable $z_{i}$ active if $z_{i}(x)=0$. The active switching set $\alpha$ consists of all indices of active switching variables,

$$
\alpha(x):=\left\{1 \leq i \leq s: z_{i}(x)=0\right\} .
$$

The numbers of active and inactive switching variables are $|\alpha(x)|$ and $|\sigma(x)|:=s-|\alpha(x)|$.

\section{LITERATURE}

Griewank and Walther have developed a class of unconstrained abs-normal problems in [3, 4]. These problems offer particularly attractive theoretical features when generalizing KKT theory and stationarity concepts to non-smooth problems. Under certain regularity conditions, they are computationally tractable by active-set type algorithms with guaranteed convergence based on piecewise linearizations and using algorithmic differentiation techniques $[5,6]$.

Another important class of non-smooth optimization problems are Mathematical Programs with Complementarity (or Equilibrium) Constraints (MPCCs, MPECs); an overview can be found in the book [12]. Since standard theory for smooth optimization problems cannot be applied, new constraint qualifications and corresponding optimality conditions were introduced. By now, there is a large body of literature on MPCCs, and we refer to [16] for an overview of the basic concepts and theory. In this paper, constraint qualifications for MPCCs in the sense of Abadie and Guignard and corresponding stationarity concepts (in particular M-stationarity and MPCC-linearized B-stationarity) are considered. Details can be found in [14], [12] and [1].

In [8] we have shown that unconstrained abs-normal problems constitute a subclass of MPCCs. In addition, we have studied regularity concepts of linear independence and of Mangasarian-Fromovitz type. As a direct generalization of unconstrained abs-normal problems we have considered NLPs with abs-normal constraints, which turned out to be equivalent to the class of MPCCs. In [10] we have extended optimality conditions of unconstrained abs-normal problems to general abs-normal NLPs under the linear independence kink qualification using a reformulation of inequalities with absolute value slacks. We have compared these optimality conditions to concepts of MPCCs in [9]. We have 
also shown that the above slack reformulation preserves kink qualifications of linear independece type but not of Mangasarian-Fromovitz type. More details and additional information about these results as well as about the results in this paper can be found in [7].

\section{CONTRIBUTIONS.}

In the present article we extend our detailed comparative study of general abs-normal NLPs and MPECs, considering constraint qualifications of Abadie and Guignard type both for the standard formulation and for the reformulation with absolute value slacks. In particular, we show that constraint qualifications of Abadie type are equivalent for abs-normal NLPs and MPCCs and that they are preserved under the slack reformulation. For constraint qualifications of Guignard type we cannot prove equivalence but only certain implications. However, when considering branch problems of abs-normal NLPs and MPCCs, we again obtain equivalence of constraint qualifications of Abadie and Guignard type, even under the slack reformulation. Finally we introduce Mordukhovich and Bouligand stationarity concepts for abs-normal NLPs and prove first order optimality conditions using the corresponding concepts for MPCCs.

\section{STRUCTURE.}

The remainder of this article is structured as follows. In Section 2 we state the general abs-normal NLP and its reformulation with absolute value slacks that permits to dispose of inequalities. We also present the branch structure of both formulations and introduce appropriate definitions of the tangent cone and the linearized cone. Using these tools, we introduce kink qualifications in the sense of Abadie and Guignard. In terms of these two kink qualifications, we then compare the regularity of the equality-constrained form of an abs-normal NLP to the inequality-constrained one. In Section 3 we introduce counterpart MPCCs for the two formulations of abs-normal NLPs and discuss the associated MPCC-constraint qualifications, namely MPCC-ACQ and MPCC-GCQ. In Section 4 we investigate the interrelation of the regularity concepts for abs-normal NLPs and MPECs and find the situation to be more intricate than under LICQ and MFCQ discussed in [10]. Finally, in Section 5 we introduce abs-normal variants of $\mathrm{M}$-stationarity and B-stationarity as first order necessary optimality conditions for abs-normal NLPs and prove equivalence relations for the respective MPCC stationarity conditions. We conclude with Section 6.

\section{INEQUALITY AND EQUALITY CONSTRAINED FORMULATIONS}

In this section we consider two different treatments of inequality constraints for non-smooth NLPs in abs-normal form.

\subsection{GENERAL ABS-NORMAL NLPS}

Substituting the representation (ANF) of constraints in abs-normal form into the general problem (NLP), we obtain a general abs-normal NLP. Here, we use the variables $\left(t, z^{t}\right)$ instead of $(x, z)$ and analogously $\sigma^{t}(t)$ and $\alpha^{t}(t)$ instead of $\sigma(x)$ and $\alpha(x)$.

Definition 2.1 (Abs-Normal NLP). Let $D^{t}$ be an open subset of $\mathbb{R}^{n_{t}}$. A non-smooth NLP is called an abs-normal NLP if functions $f \in C^{d}\left(D^{t}, \mathbb{R}\right), c_{\mathcal{E}} \in C^{d}\left(D^{t,\left|z^{t}\right|}, \mathbb{R}^{m_{1}}\right), c_{I} \in C^{d}\left(D^{t,\left|z^{t}\right|}, \mathbb{R}^{m_{2}}\right)$, and $c_{\mathcal{Z}} \in C^{d}\left(D^{t,\left|z^{t}\right|}, \mathbb{R}^{s_{t}}\right)$ with $d \geq 1$ exist such that it reads

(I-NLP) $\quad \min _{t, z^{t}} f(t) \quad$ s.t. $\quad c_{\mathcal{E}}\left(t,\left|z^{t}\right|\right)=0, \quad c_{\mathcal{I}}\left(t,\left|z^{t}\right|\right) \geq 0, \quad c_{\mathcal{Z}}\left(t,\left|z^{t}\right|\right)-z^{t}=0$, 
where $D^{\left|z^{t}\right|}$ is open and symmetric and $\partial_{2} c \mathcal{Z}\left(x,\left|z^{t}\right|\right)$ is strictly lower triangular. The feasible set of $(\mathrm{I}-\mathrm{NLP})$ is $\mathcal{F}_{\mathrm{abs}}:=\left\{\left(t, z^{t}\right): c_{\mathcal{E}}\left(t,\left|z^{t}\right|\right)=0, c_{\mathcal{I}}\left(t,\left|z^{t}\right|\right) \geq 0, c_{\mathcal{Z}}\left(t,\left|z^{t}\right|\right)=z^{t}\right\}$.

Definition 2.2 (Active Inequality Set). Let $\left(t, z^{t}(t)\right) \in \mathcal{F}_{\text {abs. }}$. We call the inequality constraint $i \in \mathcal{I}$ active if $c_{i}\left(t,\left|z^{t}(t)\right|\right)=0$. The active set $\mathcal{A}(t)$ consists of all indices of active inequality constraints, $\mathcal{A}(t)=\left\{i \in \mathcal{I}: c_{i}\left(t,\left|z^{t}(t)\right|\right)=0\right\}$. We set $c_{\mathcal{A}}:=\left[c_{i}\right]_{i \in \mathcal{H}(t)}$ and denote the number of active inequality constraints by $|\mathcal{A}(t)|$.

With the goal of considering kink qualifications in the spirit of Abadie and Guignard, we define the tangent cone and the abs-normal-linearized cone.

Definition 2.3 (Tangent Cone and Abs-Normal-Linearized Cone for (I-NLP)). Consider a feasible point $\left(t, z^{t}\right)$ of (I-NLP). The tangent cone to $\mathcal{F}_{\text {abs }}$ at $\left(t, z^{t}\right)$ is

$$
\mathcal{T}_{\text {abs }}\left(t, z^{t}\right):=\left\{\begin{array}{l|l}
\left(\delta t, \delta z^{t}\right) & \begin{array}{l}
\exists \tau_{k} \searrow 0, \mathcal{F}_{\text {abs }} \ni\left(t_{k}, z_{k}^{t}\right) \rightarrow\left(t, z^{t}\right): \\
\tau_{k}^{-1}\left(t_{k}-t, z_{k}^{t}-z^{t}\right) \rightarrow\left(\delta t, \delta z^{t}\right)
\end{array}
\end{array}\right\} .
$$

With $\delta \zeta_{i}:=\left|\delta z_{i}^{t}\right|$ if $i \in \alpha^{t}(t)$ and $\delta \zeta_{i}:=\sigma_{i}^{t}(t) \delta z_{i}^{t}$ if $i \notin \alpha^{t}(t)$, the abs-normal-linearized cone is

$$
\mathcal{T}_{\mathrm{abs}}^{\operatorname{lin}}\left(t, z^{t}\right):=\left\{\begin{array}{l|l}
\left(\delta t, \delta z^{t}\right) & \begin{array}{c}
\partial_{1} c_{\mathcal{E}}\left(t,\left|z^{t}\right|\right) \delta t+\partial_{2} c_{\mathcal{E}}\left(t,\left|z^{t}\right|\right) \delta \zeta=0 \\
\partial_{1} c_{\mathcal{A}}\left(t,\left|z^{t}\right|\right) \delta t+\partial_{2} c_{\mathcal{A}}\left(t,\left|z^{t}\right|\right) \delta \zeta \geq 0 \\
\partial_{1} c_{\mathcal{Z}}\left(t,\left|z^{t}\right|\right) \delta t+\partial_{2} c_{\mathcal{Z}}\left(t,\left|z^{t}\right|\right) \delta \zeta=\delta z^{t}
\end{array}
\end{array}\right\} .
$$

To prove that the tangent cone is a subset of the abs-normal-linearized cone, we follow an idea from [1], where an analogous result for MPCCs was obtained. First, we need the definition of the smooth branch NLPs for (I-NLP) with their standard tangent cones and linearized cones.

Definition 2.4 (Branch NLPs for (I-NLP)). Consider a feasible point $\left(\hat{t}, \hat{z}^{t}\right)$ of (I-NLP). Choose $\sigma^{t} \in$ $\{-1,1\}^{s_{t}}$ with $\sigma^{t} \geq \sigma^{t}(\hat{t})$ and set $\Sigma^{t}=\operatorname{diag}\left(\sigma^{t}\right)$. The branch problem $\operatorname{NLP}\left(\Sigma^{t}\right)$ is defined as

$\left(\operatorname{NLP}\left(\Sigma^{t}\right)\right)$

$$
\begin{array}{ll}
\min _{t, z^{t}} f(t) \quad \text { s.t. } & c_{\mathcal{E}}\left(t, \Sigma^{t} z^{t}\right)=0, \quad c_{I}\left(t, \Sigma^{t} z^{t}\right) \geq 0, \\
& c_{\mathcal{Z}}\left(t, \Sigma^{t} z^{t}\right)-z^{t}=0, \quad \Sigma^{t} z^{t} \geq 0 .
\end{array}
$$

The feasible set of $\left(\operatorname{NLP}\left(\Sigma^{t}\right)\right)$, which always contains $\left(\hat{t}, \hat{z}^{t}\right)$, is denoted by $\mathcal{F}_{\Sigma^{t}}$.

Definition 2.5 (Tangent Cone and Linearized Cone for $\left.\left(\operatorname{NLP}\left(\Sigma^{t}\right)\right)\right)$. Given $\left(\operatorname{NLP}\left(\Sigma^{t}\right)\right)$, consider a feasible point $\left(t, z^{t}\right)$. The tangent cone to $\mathcal{F}_{\Sigma^{t}}$ at $\left(t, z^{t}\right)$ is

$$
\mathcal{T}_{\Sigma^{t}}\left(t, z^{t}\right):=\left\{\begin{array}{l|l}
\left(\delta t, \delta z^{t}\right) & \begin{array}{l}
\exists \tau_{k} \searrow 0, \mathcal{F}_{\Sigma^{t}} \ni\left(t_{k}, z_{k}^{t}\right) \rightarrow\left(t, z^{t}\right): \\
\tau_{k}^{-1}\left(t_{k}-t, z_{k}^{t}-z^{t}\right) \rightarrow\left(\delta t, \delta z^{t}\right)
\end{array}
\end{array}\right\} .
$$

The linearized cone is

$$
\mathcal{T}_{\Sigma^{t}}^{\operatorname{lin}}\left(t, z^{t}\right):=\left\{\begin{array}{l|l}
\left(\delta t, \delta z^{t}\right) & \begin{array}{c}
\partial_{1} c_{\mathcal{E}}\left(t, \Sigma^{t} z^{t}\right) \delta t+\partial_{2} c_{\mathcal{E}}\left(t, \Sigma^{t} z^{t}\right) \Sigma^{t} \delta z^{t}=0 \\
\partial_{1} c_{\mathcal{A}}\left(t, \Sigma^{t} z^{t}\right) \delta t+\partial_{2} c_{\mathcal{A}}\left(t, \Sigma^{t} z^{t}\right) \Sigma^{t} \delta z^{t} \geq 0 \\
\partial_{1} c_{\mathcal{Z}}\left(t, \Sigma^{t} z^{t}\right) \delta t+\partial_{2} c_{\mathcal{Z}}\left(t, \Sigma^{t} z^{t}\right) \Sigma^{t} \delta z^{t}=\delta z^{t} \\
\sigma_{i}^{t} \delta z_{i}^{t} \geq 0, i \in \alpha^{t}(t)
\end{array}
\end{array}\right\} .
$$

Remark 2.6. Observe that $\left|z^{t}\right|=\Sigma^{t} z^{t}$ in Definition 2.4 and Definition 2.5, and for every $\Sigma^{t}$ we have $\mathcal{F}_{\Sigma^{t}} \subseteq \mathcal{F}_{\text {abs }}, \mathcal{T}_{\Sigma^{t}}\left(t, z^{t}\right) \subseteq \mathcal{T}_{\text {abs }}\left(t, z^{t}\right)$, and $\mathcal{T}_{\Sigma^{t}}^{\operatorname{lin}}\left(t, z^{t}\right) \subseteq \mathcal{T}_{\text {abs }}^{\operatorname{lin}}\left(t, z^{t}\right)$

Lemma 2.7. Consider a feasible point $\left(\hat{t}, \hat{z}^{t}\right)$ of (I-NLP) with associated branch problems $\left(\operatorname{NLP}\left(\Sigma^{t}\right)\right)$. Then, the following decompositions of the tangent cone and of the abs-normal-linearized cone of (I-NLP) hold:

$$
\mathcal{T}_{\mathrm{abs}}\left(\hat{t}, \hat{z}^{t}\right)=\bigcup_{\Sigma^{t}} \mathcal{T}_{\Sigma^{t}}\left(\hat{t}, \hat{z}^{t}\right) \quad \text { and } \quad \mathcal{T}_{\mathrm{abs}}^{\operatorname{lin}}\left(\hat{t}, \hat{z}^{t}\right)=\bigcup_{\Sigma^{t}} \mathcal{T}_{\Sigma^{t}}^{\operatorname{lin}}\left(\hat{t}, \hat{z}^{t}\right)
$$


Proof. We first consider the tangent cones and show that a neighborhood $\mathcal{N}$ of $\left(\hat{t}, \hat{z}^{t}\right)$ exists such that

$$
\mathcal{F}_{\text {abs }} \cap \mathcal{N}=\bigcup_{\Sigma^{t}}\left(\mathcal{F}_{\Sigma^{t}} \cap \mathcal{N}\right)
$$

The inclusion $\supseteq$ holds for every neighborhood $\mathcal{N}$ since $\mathcal{F}_{\Sigma^{t}} \subseteq \mathcal{F}_{\text {abs }}$ for all $\Sigma^{t}$. To show the inclusion $\subseteq$ we consider an index $i \notin \alpha^{t}(\hat{t})$. Then, by continuity, $\epsilon_{i}>0$ exists with $\sigma_{i}^{t}(t)=\sigma_{i}^{t}(\hat{t}) \in\{-1,+1\}$ for all $t \in B_{\epsilon_{i}}(\hat{t})$. Now set $\epsilon:=\min _{i \notin \alpha^{t}(\hat{t})} \epsilon_{i}, \mathcal{N}:=B_{\epsilon} \times \mathbb{R}^{n_{t}}$, and consider $\left(t, z^{t}\right) \in \mathcal{N} \cap \mathcal{F}_{\text {abs }}$. With the choice $\sigma_{i}^{t}=\sigma_{i}^{t}(t)$ for $i \notin \alpha^{t}(t)$ and $\sigma_{i}^{t}=1$ for $i \in \alpha^{t}(t)$ we find $\Sigma^{t}=\operatorname{diag}\left(\sigma^{t}\right)$ such that $\left(t, z^{t}\right) \in \mathcal{N} \cap \mathcal{F}_{\Sigma^{t}}$ since $\alpha^{t}(t) \subseteq \alpha^{t}(\hat{t})$. Thus,

$$
\mathcal{F}_{\text {abs }} \cap \mathcal{N}=\bigcup_{\Sigma^{t}}\left(\mathcal{F}_{\Sigma^{t}} \cap \mathcal{N}\right) .
$$

Now, let $\mathcal{T}\left(\hat{t}, \hat{z}^{t} ; \mathcal{F}\right)$ generically denote the tangent cone to $\mathcal{F}$ at $\left(\hat{t}, \hat{z}^{t}\right)$. Then,

$$
\begin{aligned}
\mathcal{T}_{\text {abs }}\left(\hat{t}, \hat{z}^{t}\right)=\mathcal{T}\left(\hat{t}, \hat{z}^{t} ; \mathcal{F}_{\text {abs }}\right) & =\mathcal{T}\left(\hat{t}, \hat{z}^{t} ; \mathcal{F}_{\text {abs }} \cap \mathcal{N}\right)=\mathcal{T}\left(\hat{t}, \hat{z}^{t} ; \bigcup_{\Sigma^{t}}\left(\mathcal{F}_{\Sigma^{t}} \cap \mathcal{N}\right)\right) \\
& =\bigcup_{\Sigma^{t}} \mathcal{T}\left(\hat{t}, \hat{z}^{t} ; \mathcal{F}_{\Sigma^{t}} \cap \mathcal{N}\right)=\bigcup_{\Sigma^{t}} \mathcal{T}\left(\hat{t}, \hat{z}^{t} ; \mathcal{F}_{\Sigma^{t}}\right)=\bigcup_{\Sigma^{t}} \mathcal{T}_{\Sigma^{t}}\left(\hat{t}, \hat{z}^{t}\right) .
\end{aligned}
$$

Here the fourth equality holds since the number of branch problems is finite. The decomposition of $\mathcal{T}_{\text {abs }}^{\text {lin }}$ follows directly by comparing definitions of $\mathcal{T}_{\text {abs }}^{\text {lin }}$ and $\mathcal{T}_{\Sigma^{t}}^{\text {lin }}$.

Lemma 2.8. Let $\left(t, z^{t}\right)$ be feasible for (I-NLP). Then,

$$
\mathcal{T}_{\mathrm{abs}}\left(t, z^{t}\right) \subseteq \mathcal{T}_{\mathrm{abs}}^{\operatorname{lin}}\left(t, z^{t}\right) \quad \text { and } \quad \mathcal{T}_{\mathrm{abs}}\left(t, z^{t}\right)^{*} \supseteq \mathcal{T}_{\mathrm{abs}}^{\operatorname{lin}}\left(t, z^{t}\right)^{*}
$$

Proof. The branch NLPs are smooth, hence the inclusion $\mathcal{T}_{\Sigma^{t}}\left(t, z^{t}\right) \subseteq \mathcal{T}_{\Sigma^{t}}^{\text {lin }}\left(t, z^{t}\right)$ holds by standard NLP theory. Then, the first inclusion follows directly from Lemma 2.7 and the second inclusion follows by dualization of the cones.

In general, the reverse inclusions do not hold. This leads to the following definitions.

Definition 2.9 (Abadie's and Guignard's Kink Qualifications for (I-NLP)). Consider a feasible point $\left(t, z^{t}(t)\right)$ of (I-NLP). We say that Abadie's Kink Qualification (AKQ) holds at $t$ if we have $\mathcal{T}_{\text {abs }}\left(t, z^{t}(t)\right)=$ $\mathcal{T}_{\text {abs }}^{\operatorname{lin}}\left(t, z^{t}(t)\right)$, and that Guignard's Kink Qualification (GKQ) holds at $t$ if $\mathcal{T}_{\text {abs }}\left(t, z^{t}(t)\right)^{*}=\mathcal{T}_{\text {abs }}^{\operatorname{lin}}\left(t, z^{t}(t)\right)^{*}$

The decomposition of cones in Lemma 2.7 and its dualization immediately lead to the next theorem. Theorem 2.10 (ACQ/GCQ for all (NLP $\left(\Sigma^{t}\right)$ ) implies AKQ/GKQ for (I-NLP)). Consider a feasible point $\left(t, z^{t}(t)\right)$ of (I-NLP) with associated branch problems $\left(\mathrm{NLP}\left(\Sigma^{t}\right)\right)$. Then, AKQ respectively GKQ holds for (I-NLP) at $t$ if ACQ respectively GCQ holds for all $\left(\mathrm{NLP}\left(\Sigma^{t}\right)\right)$ at $\left(t, z^{t}(t)\right)$.

\subsection{ABS-NORMAL NLPS WITH INEQUALITY SLACKS}

Here, we use absolute values of slack variables to get rid of the inequality constraints. This idea is due to Griewank. It has been introduced in [10] and has been further investigated in [9]. With slack variables $w \in \mathbb{R}^{m_{2}}$, we reformulate (NLP) as follows:

$$
\min _{t, w} f(t) \quad \text { s.t. } \quad g(t)=0, \quad h(t)-|w|=0 .
$$

Then, we express $g$ and $h$ in abs-normal form as in (ANF) and introduce additional switching variables $z^{w}$ to handle $|w|$. We obtain a class of purely equality-constrained abs-normal NLPs. 
Definition 2.11 (Abs-Normal NLP with Inequality Slacks). An abs-normal NLP posed in the following form is called an abs-normal NLP with inequality slacks:

$$
\begin{array}{lll}
\min _{t, w, z^{t}, z^{w}} f(t) \quad \text { s.t. } & c_{\mathcal{E}}\left(t,\left|z^{t}\right|\right)=0, \quad c_{\mathcal{I}}\left(t,\left|z^{t}\right|\right)-\left|z^{w}\right|=0 \\
& c_{\mathcal{Z}}\left(t,\left|z^{t}\right|\right)=z^{t}, \quad w=z^{w}
\end{array}
$$

where $D^{\left|z^{t}\right|}$ is open and symmetric and $\partial_{2} c_{Z}\left(t,\left|z^{t}\right|\right)$ is strictly lower triangular. The feasible set of $(\mathrm{E}-\mathrm{NLP})$ is denoted by $\mathcal{F}_{\text {e-abs }}$ and is a lifting of $\mathcal{F}_{\text {abs }}$.

Remark 2.12. Introducing $|w|$ converts inequalities to pure equalities without a nonnegativity condition for the slack variables $w$. In [10] we have used this formulation to simplify the presentation of first and second order conditions for the general abs-normal NLP under the linear independence kink qualification (LIKQ). Later we will see that constraint qualifications of Abadie type are preserved under reformulation. Nevertheless, this representation causes some problems. In [9] we have shown that, in contrast to LIKQ, constraint qualifications of Mangasarian-Fromovitz type are not preserved. Moreover, we cannot prove compatibility of constraint qualifications of Guignard type. Also, note that the equation $w-z^{w}=0$ (and hence $w$ ) cannot be eliminated as this would destroy the abs-normal form. Finally, the signs of nonzero components $w_{i}$ can be chosen arbitrarily and thus the slack $w$ is not uniquely determined. This needs to be taken into account when formulationg kink qualifications (KQ) for (E-NLP).

We are now interested in deriving Abadie's and Guignard's KQ for (E-NLP). To this end, we observe that the formulation (E-NLP) can be seen as a special case of (I-NLP): Let $x=(t, w), z=\left(z^{t}, z^{w}\right)$, $\bar{f}(x)=f(t), \bar{c}_{\mathcal{E}}(x,|z|)=\left(c_{\mathcal{E}}\left(t,\left|z^{t}\right|\right), c_{\mathcal{I}}\left(t,\left|z^{t}\right|\right)-\left|z^{w}\right|\right)$, and $\bar{c}_{\mathcal{Z}}(x,|z|)=\left(c_{\mathcal{Z}}\left(t,\left|z^{t}\right|\right), w\right)$. Then, we can rewrite (E-NLP) as

$$
\min _{x, z} f(x) \quad \text { s.t. } \quad \bar{c}_{\mathcal{E}}(x,|z|)=0, \quad \bar{c}_{\mathcal{Z}}(x,|z|)-z=0 .
$$

Hence, the following material is readily obtained by specializing the definitions and results in the previous section.

With $\delta=\left(\delta t, \delta w, \delta z^{t}, \delta z^{w}\right)$, Definition 2.3 and $w=z^{w}$ give the tangent cone to $\mathcal{F}_{\text {e-abs }}$ at $\left(t, w, z^{t}, z^{w}\right)$ as

$$
\mathcal{T}_{\mathrm{e} \text {-abs }}\left(t, w, z^{t}, z^{w}\right)=\left\{\begin{array}{l|l}
\delta & \begin{array}{l}
\exists \tau_{k} \searrow 0, \mathcal{F}_{\mathrm{e}-\mathrm{abs}} \ni\left(t_{k}, w_{k}, z_{k}^{t}, z_{k}^{w}\right) \rightarrow\left(t, w, z^{t}, z^{w}\right): \\
\tau_{k}^{-1}\left(t_{k}-t, w_{k}-w, z_{k}^{t}-z^{t}\right) \rightarrow\left(\delta t, \delta w, \delta z^{t}\right), \delta z^{w}=\delta w
\end{array}
\end{array}\right\},
$$

and the abs-normal-linearized cone reads

$$
\mathcal{T}_{\mathrm{e}-\mathrm{abs}}^{\operatorname{lin}}\left(t, w, z^{t}, z^{w}\right)=\left\{\begin{array}{ll}
\delta & \begin{array}{l}
\partial_{1} c_{\mathcal{I}}\left(t,\left|z^{t}\right|\right) \delta t+\partial_{2} c_{\mathcal{I}}\left(t,\left|z^{t}\right|\right) \delta \zeta=\delta \omega, \\
\left(\delta t, \delta z^{t}\right) \in \mathcal{T}_{\mathrm{abs}}^{\operatorname{lin}}\left(t, z^{t}\right), \delta z^{w}=\delta w
\end{array}
\end{array}\right\},
$$

where $\alpha=\left(\alpha^{t}, \alpha^{w}\right)$ and

$$
\delta \zeta_{i}=\left\{\begin{array}{ll}
\sigma_{i}^{t}(t) \delta z_{i}^{t}, & i \notin \alpha^{t}(t) \\
\left|\delta z_{i}^{t}\right|, & i \in \alpha^{t}(t)
\end{array}\right\}, \quad \delta \omega_{i}=\left\{\begin{array}{ll}
\sigma_{i}^{w}(w) \delta z_{i}^{w}, & i \notin \alpha^{w}(w) \\
\left|\delta z_{i}^{w}\right|, & i \in \alpha^{w}(w)
\end{array}\right\} .
$$

In Definition 2.4, consider a feasible point $\left(\hat{t}, \hat{w}, \hat{z}^{t}, \hat{z}^{w}\right)$ of (E-NLP). Choose $\sigma^{t} \in\{-1,1\}^{s_{t}}$ with $\sigma^{t} \geq$ $\sigma^{t}(\hat{t})$ and $\sigma^{w} \in\{-1,1\}^{m_{2}}$ with $\sigma^{w} \geq \sigma^{w}(\hat{w})$. Set $\Sigma^{t}=\operatorname{diag}\left(\sigma^{t}\right)$ and $\Sigma^{w}=\operatorname{diag}\left(\sigma^{w}\right)$. Then, the branch problem $\operatorname{NLP}\left(\Sigma^{t, w}\right)$ for $\Sigma^{t, w}:=\operatorname{diag}\left(\Sigma^{t}, \Sigma^{w}\right)$ reads

$\left(\operatorname{NLP}\left(\Sigma^{t, w}\right)\right)$

$$
\begin{array}{ll}
\min _{t, w, z^{t}, z^{w}} f(t) \quad \text { s.t. } \quad & c_{\mathcal{E}}\left(t, \Sigma^{t} z^{t}\right)=0, \quad c_{I}\left(t, \Sigma^{t} z^{t}\right)-\Sigma^{w} z^{w}=0, \\
& c_{\mathcal{Z}}\left(t, \Sigma^{t} z^{t}\right)-z^{t}=0, \quad w-z^{w}=0, \\
& \Sigma^{t} z^{t} \geq 0, \quad \Sigma^{w} z^{w} \geq 0 .
\end{array}
$$


The feasible set of $\left(\operatorname{NLP}\left(\Sigma^{t, w}\right)\right)$, which always contains $\left(\hat{t}, \hat{w}, \hat{z}^{t}, \hat{z}^{w}\right)$, is denoted by $\mathcal{F}_{\Sigma^{t, w}}$ and is a lifting of $\mathcal{F}_{\Sigma^{t}}$. By Definition 2.5, the tangent cone to $\mathcal{F}_{\Sigma^{t, w}}$ at $\left(t, w, z^{t}, z^{w}\right)$ reads

$$
\mathcal{T}_{\Sigma^{t, w}}\left(t, w, z^{t}, z^{w}\right)=\left\{\begin{array}{l|l}
\delta & \begin{array}{l}
\exists \tau_{k} \searrow 0, \mathcal{F}_{\Sigma^{t, w}} \ni\left(t_{k}, w_{k}, z_{k}^{t}, z_{k}^{w}\right) \rightarrow\left(t, w, z^{t}, z^{w}\right): \\
\tau_{k}^{-1}\left(t_{k}-t, w_{k}-w, z_{k}^{t}-z^{t}\right) \rightarrow\left(\delta t, \delta w, \delta z^{t}\right), \delta z^{w}=\delta w
\end{array}
\end{array}\right\}
$$

with $\delta=\left(\delta t, \delta w, \delta z^{t}, \delta z^{w}\right)$. The linearized cone reads

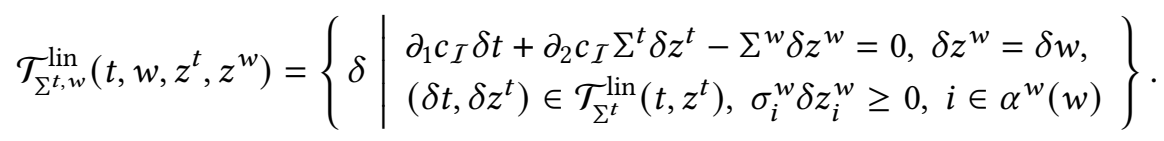

Here, all partial derivatives are evaluated at $\left(t, \Sigma^{t} z^{t}\right)$.

Moreover, we obtain the following decompositions by applying Lemma 2.7 to (E-NLP) at $y=$ $\left(t, w, z^{t}, z^{w}\right)$ with associated branch problems $\left(\operatorname{NLP}\left(\Sigma^{t, w}\right)\right)$ :

$$
\mathcal{T}_{\mathrm{e}-\mathrm{abs}}(y)=\bigcup_{\Sigma^{t, w}} \mathcal{T}_{\Sigma^{t, w}}(y) \quad \text { and } \quad \mathcal{T}_{\mathrm{e}-\mathrm{abs}}^{\operatorname{lin}}(y)=\bigcup_{\Sigma^{t, w}} \mathcal{T}_{\Sigma^{t, w}}^{\operatorname{lin}}(y)
$$

As before, the tangent cone is a subset of the linearized cone and the reverse inclusion holds for the dual cones:

$$
\mathcal{T}_{\text {e-abs }}(y) \subseteq \mathcal{T}_{\mathrm{e} \text {-abs }}^{\operatorname{lin}}(y) \quad \text { and } \quad \mathcal{T}_{\mathrm{e}-\mathrm{abs}}(y)^{*} \supseteq \mathcal{T}_{\mathrm{e} \text {-abs }}^{\operatorname{lin}}(y)^{*}
$$

This follows directly by applying Lemma 2.8 to (E-NLP). Again, equality does not hold in general, and we consider Abadie's Kink Qualification (AKQ) and Guignard's Kink Qualification (GKQ) for (E-NLP).

Given a feasible point $y=\left(t, w, z^{t}(t), z^{w}(w)\right)$ of (E-NLP), Definition 2.9 gives AKQ and GKQ at $(t, w)$, respectively, as

$$
\mathcal{T}_{\text {e-abs }}(y)=\mathcal{T}_{\mathrm{e}-\mathrm{abs}}^{\operatorname{lin}}(y) \quad \text { and } \quad \mathcal{T}_{\mathrm{e} \text {-abs }}(y)^{*}=\mathcal{T}_{\mathrm{e} \text {-abs }}^{\text {lin }}(y)^{*}
$$

Remark 2.13. The possible slack values $w \in W(t):=\left\{w:|w|=c_{\mathcal{I}}\left(t,\left|z^{t}(t)\right|\right)\right\}$ just differ by the signs of components $w_{i}$ for $i \in \mathcal{A}(t)$. Thus, neither AKQ nor GKQ depends on the particular choice of $w$, and both conditions are well-defined for (E-NLP).

Now Theorem 2.10 takes the following form.

Theorem 2.14 (ACQ/GCQ for all (NLP( $\left(\Sigma^{t, w}\right)$ ) implies AKQ/GKQ for (E-NLP)). Consider a feasible point $y=\left(t, w, z^{t}(t), z^{w}(w)\right)$ of (E-NLP) with associated branch problems $\left(\mathrm{NLP}\left(\Sigma^{t, w}\right)\right)$. Then, AKQ respectively GKQ for (E-NLP) holds at $(t, w)$ if ACQ respectively GCQ holds for all $\left(\mathrm{NLP}\left(\Sigma^{t, w}\right)\right)$ at $y$.

\subsection{RELATIONS OF KINK QUALIFICATIONS FOR ABS-NORMAL NLPS}

In this paragraph we discuss the relations of kink qualifications for the two different formulations introduced above. Here, equality of the cones and of the dual cones just needs to be considered for one element of the set $W(t)=\left\{w:|w|=c_{I}\left(t,\left|z^{t}(t)\right|\right)\right\}$. Then, it holds directly for all other elements by Remark 2.13.

Theorem 2.15. AKQ for (I-NLP) holds at $\left(t, z^{t}(t)\right) \in \mathcal{F}_{\mathrm{abs}}$ if and only if AKQ for (E-NLP) holds at $\left(t, w, z^{t}(t), z^{w}(w)\right) \in \mathcal{F}_{\mathrm{e}-\mathrm{abs}}$ for any (and hence all) $w \in W(t)$.

Proof. As $\mathcal{T}_{\text {abs }}\left(t, z^{t}\right) \subseteq \mathcal{T}_{\text {abs }}^{\text {lin }}\left(t, z^{t}\right)$ and $\mathcal{T}_{\text {eabs }}\left(t, z^{t}\right) \subseteq \mathcal{T}_{\text {e-abs }}^{\text {lin }}\left(t, z^{t}\right)$ always hold, we just need to prove

$$
\mathcal{T}_{\mathrm{abs}}\left(t, z^{t}\right) \supseteq \mathcal{T}_{\mathrm{abs}}^{\mathrm{lin}}\left(t, z^{t}\right) \Longleftrightarrow \mathcal{T}_{\mathrm{e} \text {-abs }}\left(t, w, z^{t}, z^{w}\right) \supseteq \mathcal{T}_{\mathrm{e} \text {-abs }}^{\text {lin }}\left(t, w, z^{t}, z^{w}\right)
$$


We start with the implication " $\Rightarrow$ ". Let $\delta=\left(\delta t, \delta w, \delta z^{t}, \delta z^{w}\right) \in \mathcal{T}_{\text {e-abs }}^{\operatorname{lin}}\left(t, w, z^{t}, z^{w}\right)$. Then, we have $\tilde{\delta}=\left(\delta t, \delta z^{t}\right) \in \mathcal{T}_{\text {abs }}^{\text {lin }}\left(t, z^{t}\right)=\mathcal{T}_{\text {abs }}\left(t, z^{t}\right)$. Hence, there exist sequences $\left(t_{k}, z_{k}^{t}\right) \in \mathcal{F}_{\text {abs }}$ and $\tau_{k} \searrow 0$ with $\left(t_{k}, z_{k}^{t}\right) \rightarrow\left(t, z^{t}\right)$ and $\tau_{k}^{-1}\left(t_{k}-t, z_{k}^{t}-z^{t}\right) \rightarrow\left(\delta t, \delta z^{t}\right)$. Now, define

$$
\Sigma^{w}=\operatorname{diag}(\sigma) \quad \text { with } \quad \sigma_{i}= \begin{cases}\sigma^{w}\left(w_{i}\right), & i \notin \alpha^{w}(w), \\ \operatorname{sign}\left(\delta z_{i}^{w}\right), & i \in \alpha^{w}(w),\end{cases}
$$

and set $z_{k}^{w}:=w_{k}:=\Sigma^{w} c_{I}\left(t_{k},\left|z_{k}^{t}\right|\right)$. Then, we have $z^{w}=w=\Sigma^{w} c_{I}\left(t,\left|z^{t}\right|\right)$ and obtain

$$
\begin{aligned}
z_{k}^{w}-z^{w} & =\Sigma^{w}\left[c_{I}\left(t_{k},\left|z_{k}^{t}\right|\right)-c_{I}\left(t,\left|z^{t}\right|\right)\right] \\
& =\Sigma^{w}\left[\partial_{1} c_{I}\left(t,\left|z^{t}\right|\right)\left(t_{k}-t\right)+\partial_{2} c_{I}\left(t,\left|z^{t}\right|\right)\left(\left|z_{k}^{t}\right|-\left|z^{t}\right|\right)+o\left(\left\|\left(t_{k}-t,\left|z_{k}^{t}\right|-\left|z^{t}\right|\right)\right\|\right)\right] .
\end{aligned}
$$

Further, for $k$ large enough we have $\left|z_{k}^{t}\right|-\left|z^{t}\right|=\Sigma_{k}^{t} z_{k}^{t}-\Sigma^{t} z^{t}$ using $\Sigma_{k}^{t}=\operatorname{diag}\left(\sigma_{k}^{t}\right)$ with $\sigma_{k}^{t}=\sigma\left(t_{k}\right)$ and $\Sigma^{t}=\operatorname{diag}\left(\sigma^{t}\right)$ with $\sigma^{t}=\sigma(t)$. Then, we obtain for $z_{i}^{t} \neq 0$

$$
\tau_{k}^{-1}\left(\left|\left(z_{k}^{t}\right)_{i}\right|-\left|z_{i}^{t}\right|\right)=\tau_{k}^{-1} \sigma_{i}^{t}\left(\left(z_{k}^{t}\right)_{i}-z_{i}^{t}\right) \rightarrow \sigma_{i}^{t} \delta z_{i}^{t} .
$$

For $z_{i}^{t}=0$ we have $\tau_{k}^{-1}\left(z_{k}^{t}\right)_{i} \rightarrow \delta z_{i}^{t}$ and hence

$$
\tau_{k}^{-1}\left(\left|\left(z_{k}^{t}\right)_{i}\right|-\left|z_{i}^{t}\right|\right)=\tau_{k}^{-1}\left|\left(z_{k}^{t}\right)_{i}\right| \rightarrow\left|\delta z_{i}^{t}\right| .
$$

Thus, $\tau_{k}^{-1}\left(\left|\left(z_{k}^{t}\right)\right|-\left|z^{t}\right|\right) \rightarrow \delta \zeta$ holds, and in total

$$
\tau_{k}^{-1}\left(z_{k}^{w}-z^{w}\right) \rightarrow \Sigma^{w}\left[\partial_{1} c_{\mathcal{I}}\left(t,\left|z^{t}\right|\right) \delta t+\partial_{2} c_{\mathcal{I}}\left(t,\left|z^{t}\right|\right) \delta \zeta\right]=\Sigma^{w} \delta \zeta=\delta z^{w} .
$$

Additionally, we obtain $\tau_{k}^{-1}\left(w_{k}-w\right) \rightarrow \delta w$ and finally $d \in \mathcal{T}_{\text {e-abs }}\left(t, w, z^{t}, z^{w}\right)$. To prove the implication “ $\Leftarrow$ ", consider $\delta=\left(\delta t, \delta z^{t}\right) \in \mathcal{T}_{\text {abs }}^{\text {lin }}\left(t, z^{t}\right)$. We define

$$
\Sigma^{w}=\operatorname{diag}(\sigma) \quad \text { with } \quad \sigma_{i}= \begin{cases} \pm 1, & i \in \mathcal{A}(t), \\ \operatorname{sign}\left(\left[\partial_{1} c_{\mathcal{I}}\left(t,\left|z^{t}\right|\right) \delta t+\partial_{2} c_{\mathcal{I}}\left(t,\left|z^{t}\right|\right) \delta \zeta\right]_{i}\right), & i \notin \mathcal{A}(t),\end{cases}
$$

and set $\delta w=\delta z^{w}=\Sigma^{w}\left[\partial_{1} c_{I}\left(t,\left|z^{t}\right|\right) \delta t+\partial_{2} c_{I}\left(t,\left|z^{t}\right|\right) \delta \zeta\right]$. Then we have $\tilde{\delta}=\left(\delta t, \delta w, \delta z^{t}, \delta z^{w}\right) \in$ $\mathcal{T}_{\text {e-abs }}^{\text {lin }}\left(t, w, z^{t}, z^{w}\right)$ for $w=z^{w}=\sum^{w} c_{I}\left(t,\left|z^{t}\right|\right)$. By assumption, $\tilde{\delta} \in \mathcal{T}_{\text {e-abs }}\left(t, w, z^{t}, z^{w}\right)$ holds, and this directly implies $\delta=\left(\delta t, \delta z^{t}\right) \in \mathcal{T}_{\text {abs }}\left(t, z^{t}\right)$.

Theorem 2.16. GKQfor (I-NLP) holds at $\left(t, z^{t}(t)\right) \in \mathcal{F}_{\text {abs }}$ if GKQ for (E-NLP) holds at $\left(t, w, z^{t}(t), z^{w}(w)\right) \in$ $\mathcal{F}_{\mathrm{e}-\mathrm{abs}}$ for any (and hence all) $w \in W(t)$.

Proof. The inclusion $\mathcal{T}_{\text {abs }}\left(t, z^{t}\right)^{*} \supseteq \mathcal{T}_{\text {abs }}^{\operatorname{lin}}\left(t, z^{t}\right)^{*}$ is always satisfied. Thus, we just have to show

$$
\mathcal{T}_{\mathrm{abs}}\left(t, z^{t}\right)^{*} \subseteq \mathcal{T}_{\mathrm{abs}}^{\operatorname{lin}}\left(t, z^{t}\right)^{*}
$$

Let $\omega=\left(\omega t, \omega z^{t}\right) \in \mathcal{T}_{\text {abs }}\left(t, z^{t}\right)^{*}$, i.e. $\omega^{T} \delta \geq 0$ for all $\delta=\left(\delta t, \delta z^{t}\right) \in \mathcal{T}_{\text {abs }}\left(t, z^{t}\right)$. Then, set $\tilde{\omega}=$ $\left(\omega t, 0, \omega z^{t}, 0\right)$ and obtain $\tilde{\omega}^{T} \tilde{\delta}=\omega^{T} \delta \geq 0$ for all $\tilde{\delta} \in \mathcal{T}_{\text {e-abs }}\left(t, w, z^{t}, z^{w}\right)$ where $w \in W(t)$ is arbitrary. By assumption, then $\tilde{\omega}^{T} \tilde{\delta} \geq 0$ for all $\tilde{\delta} \in \mathcal{T}_{\mathrm{e} \text {-abs }}^{\text {lin }}\left(t, w, z^{t}, z^{w}\right)$ holds. This implies $\omega^{T} \delta=\tilde{\omega}^{T} \tilde{\delta} \geq 0$ for all $\delta \in \mathcal{T}_{\text {abs }}^{\operatorname{lin}}\left(t, z^{t}\right)$.

The converse is unlikely to hold, but we are, at the same time, not aware of a counterexample. Next, we consider the branch problems and relations of ACQ and GCQ for all branch problems. Here, we can exploit sign information to show equivalence of GCQ for the branch problems of (I-NLP) and (E-NLP). Theorem 2.17. ACQ for $\left(\operatorname{NLP}\left(\Sigma^{t}\right)\right)$ holds at $\left(t, z^{t}(t)\right) \in \mathcal{F}_{\Sigma^{t}}$ if and only if ACQ for $\left(\mathrm{NLP}\left(\Sigma^{t, w}\right)\right)$ holds at $\left(t, w, z^{t}(t), z^{t}(w)\right) \in \mathcal{F}_{\Sigma^{t, w}}$ for any (and hence all) $w \in W(t)$. 
Proof. The proof proceeds as in Theorem 2.15 .

Theorem 2.18. GCQ for $\left(\mathrm{NLP}\left(\Sigma^{t}\right)\right)$ holds at $\left(t, z^{t}(t)\right) \in \mathcal{F}_{\Sigma^{t}}$ if and only if $G C Q$ for $\left(\mathrm{NLP}\left(\Sigma^{t, w}\right)\right)$ holds at $\left(t, w, z^{t}(t), z^{t}(w)\right) \in \mathcal{F}_{\Sigma^{t, w}}$ for any (and hence all) $w \in W(t)$.

Proof. The inclusions $\mathcal{T}_{\Sigma^{t}}\left(t, z^{t}\right)^{*} \supseteq \mathcal{T}_{\Sigma^{t}}^{\operatorname{lin}}\left(t, z^{t}\right)^{*}$ and $\mathcal{T}_{\Sigma^{t, w}}\left(t, z^{t}\right)^{*} \supseteq \mathcal{T}_{\Sigma^{t, w}}^{\operatorname{lin}}\left(t, z^{t}\right)^{*}$ are always satisfied. Thus, we just need to prove

$$
\mathcal{T}_{\Sigma^{t}}\left(t, z^{t}\right)^{*} \subseteq \mathcal{T}_{\Sigma^{t}}^{\operatorname{lin}}\left(t, z^{t}\right)^{*} \Longleftrightarrow \mathcal{T}_{\Sigma^{t, w}}\left(t, w, z^{t}, z^{w}\right)^{*} \subseteq \mathcal{T}_{\Sigma^{t, w}}^{\operatorname{lin}}\left(t, w, z^{t}, z^{w}\right)^{*}
$$

We start with the implication " $\Rightarrow$ ". Let $\omega=\left(\omega t, \omega w, \omega z^{t}, \omega z^{w}\right) \in \mathcal{T}_{\Sigma^{t, w}}\left(t, w, z^{t}, z^{w}\right)^{*}$, i.e. $\omega^{T} \delta \geq 0$ for all $\delta=\left(\delta t, \delta w, \delta z^{t} \delta z^{w}\right) \in \mathcal{T}_{\Sigma^{t, w}}\left(t, w, z^{t}, z^{w}\right)$. Set

$$
\tilde{\omega}=\left(\tilde{\omega} t, \tilde{\omega} z^{t}\right)=\left(\omega t, \omega z^{t}\right)+\left(\omega w+\omega z^{w}\right) \Sigma^{w}\left(\partial_{1} c_{I}\left(t, \Sigma^{t} z^{t}\right), \partial_{2} c_{I}\left(t, \Sigma^{t} z^{t}\right) \Sigma^{t}\right) .
$$

Then, we have $\tilde{\omega}^{T} \tilde{\delta}=\omega^{T} \delta \geq 0$ for all $\delta=\left(\delta t, \delta z^{t}\right) \in \mathcal{T}_{\Sigma^{t}}\left(t, z^{t}\right)$ and thus $\tilde{\omega} \in \mathcal{T}_{\Sigma^{t}}^{\operatorname{lin}}\left(t, z^{t}\right)$. Then, $\omega^{T} \delta \geq 0$ for all $\delta=\left(\delta t, \delta w, \delta z^{t} \delta z^{w}\right) \in \mathcal{T}_{\Sigma^{t, w}}^{\operatorname{lin}}\left(t, w, z^{t}, z^{w}\right)$ as $\omega^{T} \delta=\tilde{\omega}^{T} \tilde{\delta}$ holds. The reverse implication may be proved as shown in Theorem 2.16.

\section{COUNTERPART MPCCS}

In this section we restate the MPCC counterpart problems for the two formulations (I-NLP) and (E-NLP) and we present the relations between them.

\subsection{COUNTERPART MPCC FOR THE GENERAL ABS-NORMAL NLP}

To reformulate (I-NLP) as an MPCC, we split $z^{t}$ into its nonnegative part and the modulus of its nonpositive part, $u^{t}:=\left[z^{t}\right]^{+}:=\max \left(z^{t}, 0\right)$ and $v^{t}:=\left[z^{t}\right]^{-}:=\max \left(-z^{t}, 0\right)$. Then, we add complementarity of these two variables to replace $\left|z^{t}\right|$ by $u^{t}+v^{t}$ and $z^{t}$ itself by $u^{t}-v^{t}$.

Definition 3.1 (Counterpart MPCC of (I-NLP)). The counterpart MPCC of the non-smooth NLP (I-NLP) reads

$$
\begin{array}{cl}
\min _{t, u^{t}, v^{t}} f(t) \quad \text { s.t. } & c_{\mathcal{E}}\left(t, u^{t}+v^{t}\right)=0, \quad c_{\mathcal{I}}\left(t, u^{t}+v^{t}\right) \geq 0, \\
& c_{\mathcal{Z}}\left(t, u^{t}+v^{t}\right)-\left(u^{t}-v^{t}\right)=0, \\
& 0 \leq u^{t} \perp v^{t} \geq 0
\end{array}
$$

where $u^{t}, v^{t} \in \mathbb{R}^{s_{t}}$. The feasible set of (I-MPCC) is denoted by $\mathcal{F}_{\text {mpcc }}$.

Given an abs-normal NLP (I-NLP) and its counterpart MPCC (I-MPCC), the mapping $\phi: \mathcal{F}_{\text {mpcc }} \rightarrow$ $\mathcal{F}_{\text {abs }}$ defined by

$$
\phi\left(t, u^{t}, v^{t}\right)=\left(t, u^{t}-v^{t}\right) \quad \text { and } \quad \phi^{-1}\left(t, z^{t}\right)=\left(t,\left[z^{t}\right]^{+},\left[z^{t}\right]^{-}\right)
$$

is a homeomorphism. This result was obtained in [9, Lemma 31].

Corresponding to the active switching set in the previous section, we introduce index sets for MPCCs.

Definition 3.2 (Index Sets). We denote by $\mathcal{U}_{0}^{t}:=\left\{i \in\left\{1, \ldots, s_{t}\right\}: u_{i}^{t}=0\right\}$ the set of indices of active inequalities $u_{i}^{t} \geq 0$, and by $\mathcal{U}_{+}^{t}:=\left\{i \in\left\{1, \ldots, s_{t}\right\}: u_{i}^{t}>0\right\}$ the set of indices of inactive inequalities $u_{i}^{t} \geq 0$. Analogous definitions hold of $\mathcal{V}_{0}^{t}$ and $\mathcal{V}_{+}^{t}$. By $\mathcal{D}^{t}:=\mathcal{U}_{0}^{t} \cap \mathcal{V}_{0}^{t}$ we denote the set of indices of nonstrict (degenerate) complementarity pairs. Thus we have the partitioning $\left\{1, \ldots, s_{t}\right\}=\mathcal{U}_{+}^{t} \cup \mathcal{V}_{+}^{t} \cup \mathcal{D}^{t}$.

In order to define MPCC-CQs in the spirit of Abadie and Guignard, we introduce the tangent cone, the complementarity cone, and the MPCC-linearized cone. 
Definition 3.3 (Tangent Cone and MPCC-Linearized Cone for (I-MPCC), see [1]). Consider a feasible point $\left(t, u^{t}, v^{t}\right)$ of (I-MPCC) with associated index sets $\mathcal{U}_{+}^{t}, \mathcal{V}_{+}^{t}$ and $\mathcal{D}^{t}$. The tangent cone to $\mathcal{F}_{\text {mpcc }}$ at $\left(t, u^{t}, v^{t}\right)$ is

$$
\mathcal{T}_{\text {mpcc }}\left(t, u^{t}, v^{t}\right):=\left\{\begin{array}{l|l}
\left(\delta t, \delta u^{t}, \delta v^{t}\right) & \begin{array}{l}
\exists \tau_{k} \searrow 0, \mathcal{F}_{\text {mpcc }} \ni\left(t_{k}, u_{k}^{t}, v_{k}^{t}\right) \rightarrow\left(t, u^{t}, v^{t}\right): \\
\tau_{k}^{-1}\left(t_{k}-t, u_{k}^{t}-u^{t}, v_{k}^{t}-v^{t}\right) \rightarrow\left(\delta t, \delta u^{t}, \delta v^{t}\right)
\end{array}
\end{array}\right\} .
$$

The MPCC-linearized cone at $\left(t, u^{t}, v^{t}\right)$ is

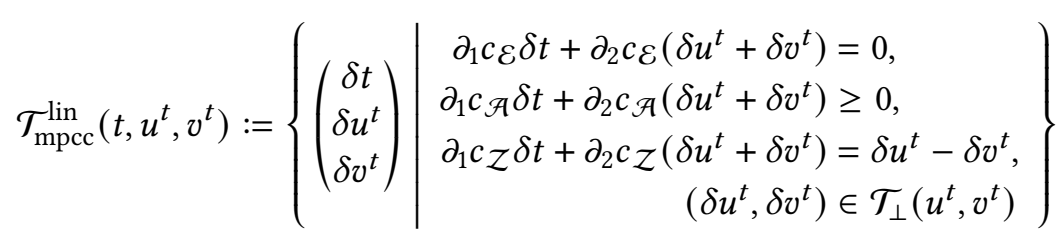

with complementarity cone

$$
\mathcal{T}_{\perp}\left(u^{t}, v^{t}\right):=\left\{\begin{array}{l|l}
\left(\delta u^{t}, \delta v^{t}\right) & \begin{array}{l}
\delta u_{i}^{t}=0, i \in \mathcal{V}_{+}^{t}, \delta v_{i}^{t}=0, i \in \mathcal{U}_{+}^{t} \\
0 \leq \delta u_{i}^{t} \perp \delta v_{i}^{t} \geq 0, i \in \mathcal{D}^{t}
\end{array}
\end{array}\right\} .
$$

Here, all partial derivatives are evaluated at $\left(t, u^{t}+v^{t}\right)$.

Note that the MPCC-linearized cone was originally stated in [11] and [14], but was not further investigated there. Moreover, we modified the definition in [1] by introducing the complementarity cone which is studied in the next lemma.

Lemma 3.4. The complementarity cone $\mathcal{T}_{\perp}\left(\hat{u}^{t}, \hat{v}^{t}\right)$ is the tangent cone and also the linearized cone to the complementarity set $\left\{\left(u^{t}, v^{t}\right): 0 \leq u^{t} \perp v^{t} \geq 0\right\}$ at $\left(\hat{u}^{t}, \hat{v}^{t}\right)$.

Proof. Given a tangent vector $\left(\delta u^{t}, \delta v^{t}\right)=\lim \tau_{k}^{-1}\left(u_{k}^{t}-\hat{u}^{t}, v_{k}^{t}-\hat{v}^{t}\right)$ where $0 \leq u_{k}^{t} \perp v_{k}^{t} \geq 0$ and $\tau_{k} \searrow 0$, we have for $k$ large enough:

$$
\begin{array}{cl}
u_{k i}^{t}>0, v_{k i}^{t}=0, & i \in \mathcal{U}_{+}^{t}\left(\hat{u}_{i}^{t}>0, \hat{v}_{i}^{t}=0\right), \\
u_{k i}^{t}=0, v_{k i}^{t}>0, & i \in \mathcal{V}_{+}^{t}\left(\hat{u}_{i}^{t}=0, \hat{v}_{i}^{t}>0\right), \\
0 \leq u_{k i}^{t} \perp v_{k i}^{t} \geq 0, & i \in \mathcal{D}^{t}\left(\hat{u}_{i}^{t}=0, \hat{v}_{i}^{t}=0\right) .
\end{array}
$$

This implies $\left(\delta u^{t}, \delta v^{t}\right) \in \mathcal{T}_{\perp}\left(\hat{u}^{t}, \hat{v}^{t}\right)$. Conversely, every $\left(\delta u^{t}, \delta v^{t}\right) \in \mathcal{T}_{\perp}\left(\hat{u}^{t}, \hat{v}^{t}\right)$ is a tangent vector generated by the sequence $\left(u_{k}^{t}, v_{k}\right)=\left(\hat{u}^{t}, \hat{v}^{t}\right)+\tau_{k}\left(\delta u^{t}, \delta v^{t}\right)$ with $\tau_{k}=1 / k, k \in \mathbb{N}_{>0}$. The linearized cone clearly coincides with the tangent cone.

Lemma 3.5. Given (I-NLP) with counterpart MPCC (I-MPCC), consider $\left(t, z^{t}\right) \in \mathcal{F}_{\text {abs }}$ with $\sigma^{t}=\sigma^{t}(t)$ and $\left(t, u^{t}, v^{t}\right)=\phi^{-1}\left(t, z^{t}\right) \in \mathcal{F}_{\text {mpcc }}$ with associated index sets $\mathcal{U}_{+}^{t}, \mathcal{V}_{+}^{t}$ and $\mathcal{D}^{t}$. Define $\psi: \mathcal{T}_{\text {mpcc }}\left(t, u^{t}, v^{t}\right) \rightarrow$ $\mathcal{T}_{\text {abs }}\left(t, z^{t}\right)$ and $\psi: \mathcal{T}_{\text {mpcc }}^{\operatorname{lin}}\left(t, u^{t}, v^{t}\right) \rightarrow \mathcal{T}_{\text {abs }}^{\text {lin }}\left(t, z^{t}\right)$ as

$$
\psi\left(\delta t, \delta u^{t}, \delta v^{t}\right)=\left(\delta t, \delta u^{t}-\delta v^{t}\right) \quad \text { and } \quad \psi^{-1}\left(\delta t, \delta z^{t}\right)=\left(\delta t,\left\langle\delta z^{t}\right\rangle^{+},\left\langle\delta z^{t}\right\rangle^{-}\right) .
$$

Here, $\left\langle\delta z^{t}\right\rangle^{+},\left\langle\delta z^{t}\right\rangle^{-}$map $\delta z^{t}$ into the complementarity cone via

$$
\left\langle\delta z_{i}^{t}\right\rangle^{+}=\left\{\begin{array}{ll}
+\delta z_{i}^{t}, & i \in \mathcal{U}_{+}^{t}\left(\sigma_{i}^{t}>0\right) \\
0, & i \in \mathcal{V}_{+}^{t}\left(\sigma_{i}^{t}<0\right) \\
{\left[\delta z_{i}^{t}\right]^{+},} & i \in \mathcal{D}^{t}\left(\sigma_{i}^{t}=0\right)
\end{array}\right\}, \quad\left\langle\delta z_{i}^{t}\right\rangle^{-}=\left\{\begin{array}{ll}
0, & i \in \mathcal{U}_{+}^{t}\left(\sigma_{i}^{t}>0\right) \\
-\delta z_{i}^{t}, & i \in \mathcal{V}_{+}^{t}\left(\sigma_{i}^{t}<0\right) \\
{\left[\delta z_{i}^{t}\right]^{-},} & i \in \mathcal{D}^{t}\left(\sigma_{i}^{t}=0\right)
\end{array}\right\} .
$$

Then, both functions $\psi$ are homeomorphisms. 
Proof. First, consider $\psi: \mathcal{T}_{\text {mpcc }}\left(t, u^{t}, v^{t}\right) \rightarrow \mathcal{T}_{\text {abs }}\left(t, z^{t}\right):$ Given a vector $\left(\delta t, \delta u^{t}, \delta v^{t}\right)=\lim \tau_{k}^{-1}\left(t_{k}-t, u_{k}^{t}-\right.$ $\left.u^{t}, v_{k}^{t}-v^{t}\right) \in \mathcal{T}_{\text {mpcc }}\left(t, u^{t}, v^{t}\right)$, set $\left(t_{k}, z_{k}^{t}\right)=\phi\left(t_{k}, u_{k}^{t}, v_{k}^{t}\right)=\left(t_{k}, u_{k}^{t}-v_{k}^{t}\right) \in \mathcal{F}_{\text {abs }}$ to obtain

$$
\lim \frac{z_{k}^{t}-z^{t}}{\tau_{k}}=\lim \frac{\left(u_{k}^{t}-u^{t}\right)-\left(v_{k}^{t}-v^{t}\right)}{\tau_{k}}=\delta u^{t}-\delta v^{t} \Longrightarrow\left(\delta t, \delta u^{t}-\delta v^{t}\right) \in \mathcal{T}_{\mathrm{abs}}\left(t, z^{t}\right) .
$$

Conversely, given a vector $\left(\delta t, \delta z^{t}\right)=\lim \tau_{k}^{-1}\left(t_{k}-t, z_{k}^{t}-z^{t}\right) \in \mathcal{T}_{\text {abs }}\left(t, z^{t}\right)$, define $\left(t_{k}, u_{k}^{t}, v_{k}^{t}\right)=\phi^{-1}\left(t_{k}, z_{k}^{t}\right)=$ $\left(t_{k},\left[z_{k}^{t}\right]^{+},\left[z_{k}^{t}\right]^{-}\right) \in \mathcal{F}_{\text {mpcc }}$. Then, $\tau_{k}^{-1}\left(\left(u_{k}-u\right)-\left(v_{k}-v\right)\right) \rightarrow\left\langle\delta z^{t}\right\rangle^{+}-\left\langle\delta z^{t}\right\rangle^{-}$holds. Thus, it remains to show $\tau_{k}^{-1}\left(u_{k}-u, v_{k}-v\right) \rightarrow\left(\left\langle\delta z^{t}\right\rangle^{+},\left\langle\delta z^{t}\right\rangle^{-}\right)$which is done componentwise:

- $i \in \mathcal{U}_{+}^{t}: v_{i}^{t}=0$ holds by feasibility and $\left\langle\delta z^{t}\right\rangle^{-}=0$ by definition. Thus, $\left(u_{k}^{t}\right)_{i}>0$ holds for $k$ large enough and by complementarity $\left(v_{k}^{t}\right)_{i}=0$ holds. Then, $\tau_{k}^{-1}\left(\left(u_{k}^{t}\right)_{i}-u_{i}^{t}\right) \rightarrow\left\langle\delta z^{t}\right\rangle_{i}^{+}$follows.

- $i \in \mathcal{V}_{+}^{t}: \tau_{k}^{-1}\left(\left(v_{k}^{t}\right)_{i}-v_{i}^{t}\right) \rightarrow\left\langle\delta z^{t}\right\rangle_{i}^{-}$follows as in the previous case.

- $i \in \mathcal{D}^{t}$ and $\left\langle\delta z^{t}\right\rangle_{i}^{+}>0:\left\langle\delta z^{t}\right\rangle_{i}^{-}=0$ holds by complementarity and so $\tau_{k}^{-1}\left(\left(u_{i}^{t}\right)_{k}-\left(v_{i}^{t}\right)_{k}\right) \rightarrow\left\langle\delta z^{t}\right\rangle_{i}^{+}$. Then, $\tau_{k}^{-1}\left(u_{i}^{t}\right)_{k} \rightarrow\left\langle\delta z^{t}\right\rangle_{i}^{+}$and $\tau_{k}^{-1}\left(v_{i}^{t}\right)_{k} \rightarrow 0$ because of sign constraints.

- $i \in \mathcal{D}^{t}$ and $\left\langle\delta z^{t}\right\rangle_{i}^{-}>0: \tau_{k}^{-1}\left(u_{i}^{t}\right)_{k} \rightarrow 0$ and $\tau_{k}^{-1}\left(v_{i}^{t}\right)_{k} \rightarrow\left\langle\delta z^{t}\right\rangle_{i}^{-}$follow as in the previous case.

- $i \in \mathcal{D}^{t}$ and $\left\langle\delta z^{t}\right\rangle_{i}^{+}=\left\langle\delta z^{t}\right\rangle_{i}^{-}=0$ : Then, $\tau_{k}^{-1}\left(\left(u_{i}^{t}\right)_{k}-\left(v_{i}^{t}\right)_{k}\right) \rightarrow 0$ holds. Because of sign constraints and complementarity, this can only hold if $\tau_{k}^{-1}\left(u_{i}^{t}\right)_{k} \rightarrow 0, \tau_{k}^{-1}\left(v_{i}^{t}\right)_{k} \rightarrow 0$.

Altogether, this implies

$$
\lim \frac{\left(t_{k}-t, u_{k}^{t}-u^{t}, v_{k}^{t}-v^{t}\right)}{\tau_{k}}=\left(\delta t,\left\langle\delta z^{t}\right\rangle^{+},\left\langle\delta z^{t}\right\rangle^{-}\right) \in \mathcal{T}_{\text {mpcc }}\left(t, u^{t}, v^{t}\right)
$$

By construction, $\psi$ and $\psi^{-1}$ are both continuous and inverse to each other.

Second, consider $\psi: \mathcal{T}_{\text {mpcc }}^{\operatorname{lin}}\left(t, u^{t}, v^{t}\right) \rightarrow \mathcal{T}_{\text {abs }}^{\operatorname{lin}}\left(t, z^{t}\right):$ Given $\left(\delta t, \delta u^{t}, \delta v^{t}\right) \in \mathcal{T}_{\text {mpcc }}^{\operatorname{lin}}\left(t, u^{t}, v^{t}\right)$, the vectors $\delta z^{t}=\delta u^{t}-\delta v^{t}$ and $\delta \zeta=\delta u^{t}+\delta v^{t}$ satisfy

$$
\delta z_{i}^{t}=\left\{\begin{array}{ll}
\delta u_{i}^{t}, & i \in \mathcal{U}_{+}^{t} \\
-\delta v_{i}^{t}, & i \in \mathcal{V}_{+}^{t} \\
\delta u_{i}^{t}-\delta v_{i}^{t}, & i \in \mathcal{D}^{t}
\end{array}\right\}, \quad \delta \zeta_{i}=\left\{\begin{array}{ll}
\delta u_{i}^{t}=\sigma_{i} \delta z_{i}^{t}, & i \in \mathcal{U}_{+}^{t} \\
\delta v_{i}^{t}=\sigma_{i}^{t} \delta z_{i}^{t}, & i \in \mathcal{V}_{+}^{t} \\
\delta u_{i}^{t}+\delta v_{i}^{t}=\left|\delta z_{i}^{t}\right|, & i \in \mathcal{D}^{t}
\end{array}\right\} .
$$

Thus, $\left(\delta t, \delta z^{t}\right)=\psi\left(\delta t, \delta u^{t}, \delta v^{t}\right) \in \mathcal{T}_{\text {abs }}^{\operatorname{lin}}\left(t, z^{t}\right)$.

Conversely, the same case distinction yields $\left(\delta t, \delta u^{t}, \delta v^{t}\right)=\psi^{-1}\left(\delta t, \delta z^{t}\right) \in \mathcal{T}_{\text {mpcc }}^{\operatorname{lin}}\left(\hat{t}, \hat{u}^{t}, \hat{v}^{t}\right)$ for every $\left(\delta t, \delta z^{t}\right) \in \mathcal{T}_{\text {abs }}^{\operatorname{lin}}\left(\hat{t}, \hat{z}^{t}\right)$. Again, $\psi$ and $\psi^{-1}$ are both continuous and inverse to each other by construction.

Definition 3.6 (Branch NLPs for (I-MPCC), see [11]). Consider a feasible point $\left(\hat{t}, \hat{u}^{t}, \hat{v}^{t}\right)$ of (I-MPCC) with associated index sets $\mathcal{U}_{+}^{t}, \mathcal{V}_{+}^{t}$, and $\mathcal{D}^{t}$ and choose $\mathcal{P}^{t} \subseteq \mathcal{D}^{t}$ with complement $\overline{\mathcal{P}}^{t}=\mathcal{D}^{t} \backslash \mathcal{P}^{t}$. The branch problem $\operatorname{NLP}\left(\mathcal{P}^{t}\right)$ is defined as

$\left(\operatorname{NLP}\left(\mathcal{P}^{t}\right)\right)$

$$
\begin{array}{cl}
\min _{t, u^{t}, v^{t}} f(t) \quad \text { s.t. } & c_{\mathcal{E}}\left(t, u^{t}+v^{t}\right)=0, \\
& c_{\mathcal{I}}\left(t, u^{t}+v^{t}\right) \geq 0, \\
& c_{\mathcal{Z}}\left(t, u^{t}+v^{t}\right)-\left(u^{t}-v^{t}\right)=0, \\
& 0=u_{i}^{t}, 0 \leq v_{i}^{t}, i \in \mathcal{V}_{+}^{t} \cup \mathcal{P}^{t}, \\
& 0 \leq u_{i}^{t}, 0=v_{i}^{t}, i \in \mathcal{U}_{+}^{t} \cup \overline{\mathcal{P}}^{t} .
\end{array}
$$

The feasible set of $\left(\operatorname{NLP}\left(\mathcal{P}^{t}\right)\right)$, which always contains $\left(\hat{t}, \hat{u}^{t}, \hat{v}^{t}\right)$, is denoted by $\mathcal{F}_{\mathcal{P} t}$. 
Clearly, the homeomorphism $\phi$ can be restricted to the branch problems $\left(\operatorname{NLP}\left(\Sigma^{t}\right)\right)$ and $\left(\operatorname{NLP}\left(\mathcal{P}^{t}\right)\right)$ where $\mathcal{P}^{t}=\left\{i \in \alpha^{t}(\hat{t}): \sigma_{i}^{t}=-1\right\}$. Thus, the mapping $\phi_{\mathcal{P}^{t}}: \mathcal{F}_{\mathcal{P} t} \rightarrow \mathcal{F}_{\Sigma^{t}}$ defined by

$$
\phi_{\mathcal{P} t}:=\left.\phi\right|_{\mathcal{P} t} \quad \text { and } \quad \phi_{\mathcal{P} t}^{-1}:=\left.\phi^{-1}\right|_{\Sigma^{t}}
$$

is a homeomorphism. The tangent cone to $\mathcal{F}_{\mathcal{P} t}$ at $\left(t, u^{t}, v^{t}\right)$ is

$$
\mathcal{T}_{\mathcal{P} t}\left(t, u^{t}, v^{t}\right):=\left\{\begin{array}{l|l}
\left(\delta t, \delta u^{t}, \delta v^{t}\right) & \begin{array}{l}
\exists \tau_{k} \searrow 0, \mathcal{F}_{\mathcal{P} t} \ni\left(t_{k}, u_{k}^{t}, v_{k}^{t}\right) \rightarrow\left(t, u^{t}, v^{t}\right): \\
\tau_{k}^{-1}\left(t_{k}-t, u_{k}^{t}-u^{t}, v_{k}^{t}-v^{t}\right) \rightarrow\left(\delta t, \delta u^{t}, \delta v^{t}\right)
\end{array}
\end{array}\right\} .
$$

The linearized cone is

$$
\mathcal{T}_{\mathcal{P} t}^{\operatorname{lin}}\left(t, u^{t}, v^{t}\right):=\left\{\begin{array}{l|l}
\left(\begin{array}{l}
\delta t \\
\delta u^{t} \\
\delta v^{t}
\end{array}\right) & \begin{array}{l}
\partial_{1} c_{\mathcal{E}} \delta t+\partial_{2} c_{\mathcal{E}}\left(\delta u^{t}+\delta v^{t}\right)=0 \\
\partial_{1} c_{\mathcal{P}} \delta t+\partial_{2} c_{\mathcal{A}}\left(\delta u^{t}+\delta v^{t}\right) \geq 0 \\
\partial_{1} c_{\mathcal{Z}} \delta t+\partial_{2} c_{\mathcal{Z}}\left(\delta u^{t}+\delta v^{t}\right)=\delta u^{t}-\delta v^{t} \\
0=\delta u_{i}^{t} \text { for } i \in \mathcal{V}_{+}^{t} \cup \mathcal{P}, 0=\delta v_{i}^{t} \text { for } i \in \mathcal{U}_{+}^{t} \cup \overline{\mathcal{P}}, \\
0 \leq \delta u_{i}^{t} \text { for } i \in \overline{\mathcal{P}}, 0 \leq \delta v_{i}^{t} \text { for } i \in \mathcal{P}
\end{array}
\end{array}\right\} .
$$

Here, all partial derivatives are evaluated at $\left(t, u^{t}+v^{t}\right)$.

Lemma 3.7. Given $\left(\operatorname{NLP}\left(\Sigma^{t}\right)\right)$ and $\left(\operatorname{NLP}\left(\mathcal{P}^{t}\right)\right)$ with $\mathcal{P}^{t}=\left\{i \in \alpha^{t}(\hat{t}): \sigma_{i}^{t}=-1\right\}$. Consider $\left(t, z^{t}\right) \in \mathcal{F}_{\Sigma^{t}}$ and

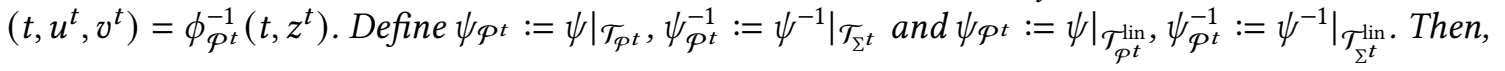

$$
\psi_{\mathcal{P}}: \mathcal{T}_{\mathcal{P}}\left(t, u^{t}, v^{t}\right) \rightarrow \mathcal{T}_{\Sigma^{t}}\left(t, \hat{z}^{t}\right) \quad \text { and } \quad \psi_{\mathcal{P}}: \mathcal{T}_{\mathcal{P} t}^{\operatorname{lin}}\left(t, \hat{u}^{t}, \hat{v}^{t}\right) \rightarrow \mathcal{T}_{\Sigma^{t}}^{\operatorname{lin}}\left(t, \hat{z}^{t}\right)
$$

are homeomorphisms.

Proof. By construction and since $\alpha^{t}(\hat{t})=\mathcal{D}^{t}$, the following equalities of sets hold:

$$
\begin{array}{ll}
\mathcal{P}^{t}=\left\{i \in \alpha^{t}(\hat{t}): \sigma_{i}^{t}=-1\right\}, & \mathcal{V}_{+}^{t}=\left\{i \notin \alpha^{t}(\hat{t}): \sigma_{i}^{t}=-1\right\}, \\
\overline{\mathcal{P}}^{t}=\left\{i \in \alpha^{t}(\hat{t}): \sigma_{i}^{t}=+1\right\}, & \mathcal{U}_{+}^{t}=\left\{i \notin \alpha^{t}(\hat{t}): \sigma_{i}^{t}=+1\right\} .
\end{array}
$$

Thus, the claim follows directly from Lemma 3.5 .

Consider a feasible point $\left(t, u^{t}, v^{t}\right)$ of (I-MPCC) with associated branch problems $\left(\operatorname{NLP}\left(\mathcal{P}^{t}\right)\right)$. Then, the following decompositions of the tangent cone and of the abs-normal-linearized cone of (I-MPCC) hold (for a proof see [1]):

$$
\mathcal{T}_{\text {mpcc }}\left(t, u^{t}, v^{t}\right)=\bigcup_{\mathcal{P} t} \mathcal{T}_{\mathcal{P} t}\left(t, u^{t}, v^{t}\right) \quad \text { and } \quad \mathcal{T}_{\text {mpcc }}^{\operatorname{lin}}\left(t, u^{t}, v^{t}\right)=\bigcup_{\mathcal{P} t} \mathcal{T}_{\mathcal{P} t}^{\operatorname{lin}}\left(t, u^{t}, v^{t}\right)
$$

The following inclusions are also proved in [1]:

$$
\mathcal{T}_{\text {mpcc }}\left(t, u^{t}, v^{t}\right) \subseteq \mathcal{T}_{\text {mpcc }}^{\operatorname{lin}}\left(t, u^{t}, v^{t}\right) \quad \text { and } \quad \mathcal{T}_{\text {mpcc }}\left(t, u^{t}, v^{t}\right)^{*} \supseteq \mathcal{T}_{\text {mpcc }}^{\operatorname{lin}}\left(t, u^{t}, v^{t}\right)^{*}
$$

In general, the converses do not hold. This motivates the definition of MPCC-ACQ and MPCC-GCQ. Definition 3.8 (Abadie's and Guignard's Constraint Qualifications for (I-MPCC), see [1]). Consider a feasible point $\left(t, u^{t}, v^{t}\right)$ of (I-MPCC). We say that Abadie's Constraint Qualification for MPCC (MPCC$A C Q)$ holds at $\left(t, u^{t}, v^{t}\right)$ if $\mathcal{T}_{\text {mpcc }}\left(t, u^{t}, v^{t}\right)=\mathcal{T}_{\text {mpcc }}^{\operatorname{lin}}\left(t, u^{t}, v^{t}\right)$, and that Guignard's Constraint Qualification for MPCC (MPCC-GCQ) holds at $\left(t, u^{t}, v^{t}\right)$ if $\mathcal{T}_{\text {mpcc }}\left(t, u^{t}, v^{t}\right)^{*}=\mathcal{T}_{\text {mpcc }}^{\operatorname{lin}}\left(t, u^{t}, v^{t}\right)^{*}$.

The decomposition (3.1) and its dualization imply that both MPCC-CQs hold if the corresponding CQ holds for all branch problems.

Theorem 3.9 (ACQ/GCQ for all (NLP( $\left.\mathcal{P}^{t}\right)$ ) implies MPCC-ACQ/MPCC-GCQ for (I-MPCC)). Consider a feasible point $\left(t, u^{t}, v^{t}\right)$ of (I-MPCC). Then, MPCC-ACQ respectively MPCC-GCQ holds at $\left(t, u^{t}, v^{t}\right)$ if $A C Q$ respectively $G C Q$ holds for all $\left(\mathrm{NLP}\left(\mathcal{P}^{t}\right)\right)$ at $\left(t, u^{t}, v^{t}\right)$. 


\subsection{COUNTERPART MPCC FOR THE ABS-NORMAL NLP WITH INEQUALITY SLACKS}

By Definition 3.1, the counterpart MPCC of the non-smooth NLP (E-NLP) reads:

(E-MPCC)

$$
\begin{array}{ll}
\min _{t, w, u^{t}, v^{t}, u^{w}, v^{w}} f(t) \quad \text { s.t. } & c_{\mathcal{E}}\left(t, u^{t}+v^{t}\right)=0, \\
& c_{\mathcal{I}}\left(t, u^{t}+v^{t}\right)-\left(u^{w}+v^{w}\right)=0, \\
& c_{\mathcal{Z}}\left(t, u^{t}+v^{t}\right)-\left(u^{t}-v^{t}\right)=0, \\
& w-\left(u^{w}-v^{w}\right)=0, \\
& 0 \leq u^{t} \perp v^{t} \geq 0, \quad 0 \leq u^{w} \perp v^{w} \geq 0,
\end{array}
$$

where $u^{t}, v^{t} \in \mathbb{R}^{s_{t}}$ and $u^{w}, v^{w} \in \mathbb{R}^{m_{2}}$. The feasible set is denoted by $\mathcal{F}_{\text {e-mpcc }}$ and is a lifting of $\mathcal{F}_{\text {mpcc }}$.

Clearly, the homeomorphism between $\mathcal{F}_{\text {mpcc }}$ and $\mathcal{F}_{\text {abs }}$ extends to $\mathcal{F}_{\text {e-mpcc }}$ and $\mathcal{F}_{\text {e-abs }}$. It is given by

$$
\begin{aligned}
\bar{\phi}\left(t, w, u^{t}, v^{t}, u^{w}, v^{w}\right) & =\left(t, w, u^{t}-v^{t}, u^{w}-v^{w}\right) \\
\bar{\phi}^{-1}\left(t, w, z^{t}, z^{w}\right) & =\left(t, w,\left[z^{t}\right]^{+},\left[z^{t}\right]^{-},\left[z^{w}\right]^{+},\left[z^{w}\right]^{-}\right) .
\end{aligned}
$$

Just like in the abs-normal case, problem (E-MPCC) is a special case of (I-MPCC). Hence, we obtain the following material by specializing the definitions and results for (I-MPCC).

By Definition 3.3, the tangent cone to $\mathcal{F}_{\text {e-mpcc }}$ at $y$ reads

$$
\mathcal{T}_{\mathrm{e}-\mathrm{mpcc}}(y)=\left\{\begin{array}{l|l}
\delta & \begin{array}{l}
\exists \tau_{k} \searrow 0, \mathcal{F}_{\mathrm{e}-\mathrm{mpcc}} \ni y_{k}=\left(t_{k}, w_{k}, u_{k}^{t}, v_{k}^{t}, u_{k}^{w}, v_{k}^{w}\right) \rightarrow y: \\
\tau_{k}^{-1}\left(y_{k}-y\right) \rightarrow \delta=\left(\delta t, \delta w, \delta u^{t}, \delta v^{t}, \delta u^{w}, \delta v^{w}\right)
\end{array}
\end{array}\right\} .
$$

The MPCC-linearized cone reads

$$
\mathcal{T}_{\mathrm{e}-\mathrm{mpcc}}^{\operatorname{lin}}(\hat{y})=\left\{\begin{array}{l|l}
\delta & \begin{array}{l}
\partial_{1} c_{\mathcal{I}} \delta t+\partial_{2} c_{\mathcal{I}}\left(\delta u^{t}+\delta v^{t}\right)=\delta u^{w}+\delta v^{w}, \delta w=\delta u^{w}-\delta v^{w} \\
\left(\delta t, \delta u^{t} \delta v^{t}\right) \in \mathcal{T}_{\operatorname{mpcc}}\left(t, u^{t}, v^{t}\right),\left(\delta u^{w}, \delta v^{w}\right) \in \mathcal{T}_{\perp}\left(\hat{u}^{w}, \hat{v}^{w}\right)
\end{array}
\end{array}\right\}
$$

Here, all partial derivatives are evaluated at $\left(t, u^{t}+v^{t}\right)$. The associated homeomorphisms of Lemma 3.5,

$$
\begin{aligned}
& \bar{\psi}: \mathcal{T}_{\mathrm{e}-\mathrm{mpcc}}\left(t, w, u^{t}, v^{t}, u^{w}, v^{w}\right) \rightarrow \mathcal{T}_{\mathrm{e}-\mathrm{abs}}\left(t, w, z^{t}, z^{w}\right), \\
& \bar{\psi}: \mathcal{T}_{\mathrm{e}-\mathrm{mpcc}}^{\operatorname{lin}}\left(t, w, u^{t}, v^{t}, u^{w}, v^{w}\right) \rightarrow \mathcal{T}_{\mathrm{e}-\mathrm{abs}}^{\operatorname{lin}}\left(t, w, z^{t}, z^{w}\right),
\end{aligned}
$$

now take the form

$$
\begin{aligned}
\bar{\psi}\left(\delta t, \delta w, \delta u^{t}, \delta v^{t}, \delta u^{w}, \delta v^{w}\right) & =\left(\delta t, \delta w, \delta u^{t}-\delta v^{t}, \delta u^{w}-\delta v^{w}\right) \\
\bar{\psi}^{-1}\left(\delta t, \delta w, \delta z^{t}, \delta z^{w}\right) & =\left(\delta t, \delta w,\left\langle\delta z^{t}\right\rangle^{+},\left\langle\delta z^{t}\right\rangle^{-},\left\langle\delta z^{w}\right\rangle^{+},\left\langle\delta z^{w}\right\rangle^{-}\right)
\end{aligned}
$$

Given $\hat{y}=\left(\hat{t}, \hat{w}, \hat{u}^{t}, \hat{v}^{t}, \hat{u}^{w}, \hat{v}^{w}\right)$, a feasible point of (E-MPCC) with associated index sets $\mathcal{U}_{+}^{t}, \mathcal{V}_{+}^{t}, \mathcal{D}^{t}$, $\mathcal{U}_{+}^{w}, \mathcal{V}_{+}^{w}$, and $\mathcal{D}^{w}$, choose $\mathcal{P}^{t} \subseteq \mathcal{D}^{t}$ as well as $\mathcal{P}^{w} \subseteq \mathcal{D}^{w}$ and set $\mathcal{P}^{t, w}=\mathcal{P}^{t} \cup \mathcal{P}^{w}$. The branch problem $\operatorname{NLP}\left(\mathcal{P}^{t, w}\right)$ of Definition 3.6 then reads

$\left(\operatorname{NLP}\left(\mathcal{P}^{t, w}\right)\right)$

$$
\begin{array}{ll}
\min _{t, w, u^{t}, v^{t}, u^{w}, v^{w}} f(t) \quad \text { s.t. } & c_{\mathcal{E}}\left(t, u^{t}+v^{t}\right)=0, \quad c_{\mathcal{I}}\left(t, u^{t}+v^{t}\right)-\left(u^{w}+v^{w}\right)=0, \\
& c_{\mathcal{Z}}\left(t, u^{t}+v^{t}\right)-\left(u^{t}-v^{t}\right)=0, \quad w-\left(u^{w}-v^{w}\right)=0, \\
& 0=u_{i}^{t}, 0 \leq v_{i}^{t}, i \in \mathcal{V}_{+}^{t} \cup \mathcal{P}^{t} \\
& 0 \leq u_{i}^{t}, 0=v_{i}^{t}, i \in \mathcal{U}_{+}^{t} \cup \overline{\mathcal{P}} t \\
& 0=u_{i}^{w}, 0 \leq v_{i}^{w}, i \in \mathcal{V}_{+}^{w} \cup \mathcal{P}^{w} \\
& 0 \leq u_{i}^{w}, 0=v_{i}^{w}, i \in \mathcal{U}_{+}^{w} \cup \mathcal{P}^{w}
\end{array}
$$

The feasible set of $\left(\operatorname{NLP}\left(\mathcal{P}^{t, w}\right)\right)$, which always contains $\hat{y}$, is denoted by $\mathcal{F}_{\mathcal{P}}$, w and is a lifting of $\mathcal{F}_{\mathcal{P} t}$. 
Again, the homeomorphism between feasible sets can be restricted to the respective branch problems $\left(\operatorname{NLP}\left(\Sigma^{t, w}\right)\right)$ and $\left(\operatorname{NLP}\left(\mathcal{P}^{t, w}\right)\right)$ where $\mathcal{P}^{t}=\left\{i \in \alpha^{t}(\hat{t}): \sigma_{i}^{t}=-1\right\}$ and $\mathcal{P}^{w}=\left\{i \in \alpha^{w}(\hat{w}): \sigma_{i}^{w}=-1\right\}$. Thus, the mapping $\bar{\phi}_{\mathcal{P} t, w}: \mathcal{F}_{\mathcal{P} t, w} \rightarrow \mathcal{F}_{\Sigma^{t, w}}$ given as

$$
\bar{\phi} \mathcal{P} t, w:=\left.\bar{\phi}\right|_{\mathcal{P} t, w} \quad \text { and } \quad \bar{\phi}_{\mathcal{P} t, w}^{-1}:=\left.\bar{\phi}^{-1}\right|_{\Sigma^{t, w}}
$$

is a homeomorphism.

The tangent cone to $\mathcal{F}_{\mathcal{P} t, w}$ at $y$ reads

$$
\mathcal{T}_{\mathcal{P} t, w}(y)=\left\{\begin{array}{l|l}
\delta & \begin{array}{l}
\exists \tau_{k} \searrow 0, \mathcal{F}_{\mathcal{P} t, w} \ni\left(t_{k}, w_{k}, u_{k}^{t}, v_{k}^{t}, u_{k}^{w}, v_{k}^{w}\right) \rightarrow\left(t, w, u^{t}, v^{t}, u^{w}, v^{w}\right): \\
\tau_{k}^{-1}\left(t_{k}-t, w_{k}-w, u_{k}^{t}-u^{t}, v_{k}^{t}-v^{t}, u_{k}^{w}-u^{w}, v_{k}^{w}-v^{w}\right) \rightarrow \delta
\end{array}
\end{array}\right\}
$$

where $\delta=\left(\delta t, \delta w, \delta u^{t}, \delta v^{t}, \delta u^{w}, \delta v^{w}\right)$. The linearized cone reads

$$
\mathcal{T}_{\mathcal{P} t, w}^{\operatorname{lin}}(y)=\left\{\begin{array}{ll}
\delta & \begin{array}{l}
\left(\delta t, \delta u^{t}, \delta v^{t}\right) \in \mathcal{T}_{\mathcal{P} t}^{\operatorname{lin}}, \\
\partial_{1} c_{I} \delta t+\partial_{2} c_{I}\left(\delta u^{t}+\delta v^{t}\right)=\delta u^{w}+\delta v^{w}, \delta w=\delta u^{w}-\delta v^{w} \\
0=\delta u_{i}^{w} \text { for } i \in \mathcal{V}_{+}^{w} \cup \mathcal{P}^{w}, 0=\delta v_{i}^{w} \text { for } i \in \mathcal{U}_{+}^{w} \cup \overline{\mathcal{P}}^{w} \\
0 \leq \delta u_{i}^{w} \text { for } i \in \mathcal{P}^{w}, 0 \leq \delta v_{i}^{w} \text { for } i \in \mathcal{P}^{w}
\end{array}
\end{array}\right\} .
$$

Here, all partial derivatives are evaluated at $\left(t, u^{t}+v^{t}\right)$. The associated cone homeomorphisms of Lemma 3.7 are now obtained as follows. Given $\left(\operatorname{NLP}\left(\Sigma^{t, w}\right)\right)$ and $\left(\operatorname{NLP}\left(\mathcal{P}^{t, w}\right)\right)$ with $\mathcal{P}^{t}=\left\{i \in \alpha^{t}(\hat{t}): \sigma_{i}^{t}=\right.$ $-1\}$ and $\mathcal{P}^{w}=\left\{i \in \alpha^{w}(\hat{w}): \sigma_{i}^{w}=-1\right\}$, consider $\left(t, w, z^{t}, z^{w}\right) \in \mathcal{F}_{\text {e-abs }}$ and $\left(t, w, u^{t}, v^{t}, u^{w}, v^{w}\right)=$

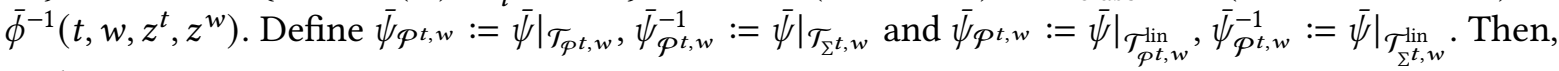
we have

$$
\begin{aligned}
& \bar{\psi}_{\mathcal{P} t, w}: \mathcal{T}_{\mathcal{P} t, w}\left(t, w, u^{t}, v^{t}, u^{w}, v^{w}\right) \rightarrow \mathcal{T}_{\Sigma^{t, w}}\left(t, w, z^{t}, z^{w}\right), \\
& \bar{\psi}_{\mathcal{P} t, w}: \mathcal{T}_{\mathcal{P} t, w}^{\operatorname{lin}}\left(t, w, u^{t}, v^{t}, u^{w}, v^{w}\right) \rightarrow \mathcal{T}_{\Sigma^{t, w}}^{\operatorname{lin}}\left(t, w, z^{t}, z^{w}\right) .
\end{aligned}
$$

By applying (3.1) to (E-MPCC) with associated branch problems $\left(\operatorname{NLP}\left(\mathcal{P}^{t, w}\right)\right)$, we obtain the following decomposition of cones at $y=\left(t, w, u^{t}, v^{t}, u^{w}, v^{w}\right)$ :

$$
\mathcal{T}_{\mathrm{e}-\mathrm{mpcc}}(y)=\bigcup_{\mathcal{P} t, w} \mathcal{T}_{\mathcal{P} t, w}(y) \quad \text { and } \quad \mathcal{T}_{\mathrm{e}-\mathrm{mpcc}}^{\operatorname{lin}}(y)=\bigcup_{\mathcal{P} t, w} \mathcal{T}_{\mathcal{P} t, w}^{\operatorname{lin}}(y) .
$$

Moreover, the tangent cone is contained in the linearized cone and the converse holds for the dual cones:

$$
\mathcal{T}_{\text {e-mpcc }}(y) \subseteq \mathcal{T}_{\mathrm{e}-\mathrm{mpcc}}^{\operatorname{lin}}(y) \quad \text { and } \quad \mathcal{T}_{\mathrm{e}-\mathrm{mpcc}}(y)^{*} \supseteq \mathcal{T}_{\mathrm{e}-\mathrm{mpcc}}^{\operatorname{lin}}(y)^{*}
$$

Once again, the converses do not hold in general and we consider Abadie's and Guignard's Constraint Qualifications for (E-MPCC) at $y=\left(t, w, u^{t}, v^{t}, u^{w}, v^{w}\right)$. Recalling Definition 3.8, MPCC-ACQ and MPCC-GCQ simply read

$$
\mathcal{T}_{\mathrm{e}-\mathrm{mpcc}}(y)=\mathcal{T}_{\mathrm{e}-\mathrm{mpcc}}^{\operatorname{lin}}(y) \quad \text { and } \quad \mathcal{T}_{\mathrm{e}-\mathrm{mpcc}}(y)^{*}=\mathcal{T}_{\mathrm{e}-\mathrm{mpcc}}^{\operatorname{lin}}(y)^{*}
$$

Remark 3.10. Let

$$
W\left(t, u^{t}, v^{t}\right)=\left\{\left(w, u^{w}, v^{w}\right):|w|=c_{I}\left(t, u^{t}+v^{t}\right), u^{w}=[w]^{+}, v^{w}=[w]^{-}\right\} .
$$

Due to symmetry, the above equality of cones (respectively dual cones) clearly holds for all elements $\left(w, u^{w}, v^{w}\right) \in W\left(t, u^{t}, v^{t}\right)$ if it holds for any element.

Now Theorem 3.9 reads as follows.

Theorem 3.11 (ACQ/GCQ for all (NLP( $\left.\mathcal{P}^{t, w}\right)$ ) implies MPCC-ACQ/MPCC-GCQ for (E-MPCC)). Consider a feasible point $y=\left(t, w, u^{t}, v^{t}, u^{w}, v^{w}\right)$ of (E-MPCC) with branch problems $\left(\mathrm{NLP}\left(\mathcal{P}^{t, w}\right)\right)$. Then, MPCC$A C Q$ respectively MPCC-GCQ holds for (E-MPCC) at y if ACQ respectively GCQ holds for all $\left(\mathrm{NLP}\left(\mathcal{P}^{t, w}\right)\right)$ at $y$. 


\subsection{RELATIONS OF MPCC-CQS FOR DIFFERENT FORMULATIONS}

In this paragraph we prove relations between constraint qualifications for the two different formulations (I-MPCC) and (E-MPCC). Some relations follow from the results in the previous section and in the two following sections.

Theorem 3.12. MPCC-ACQ for (I-MPCC) holds at $\left(t, u^{t}, v^{t}\right) \in \mathcal{F}_{\text {mpcc }}$ if and only if MPCC-ACQ for (E-MPCC) holds at $\left(t, w, u^{t}, u^{w}, v^{t}, v^{w}\right) \in \mathcal{F}_{\mathrm{e}-\mathrm{mpcc}}$ for any (and hence all) $\left(w, u^{w}, v^{w}\right) \in W\left(t, u^{t}, v^{t}\right)$.

Proof. This follows immediately from Theorem 2.15, Theorem 4.1 and Theorem 4.5 .

Theorem 3.13. MPCC-GCQ for (I-MPCC) holds at $\left(t, u^{t}, v^{t}\right) \in \mathcal{F}_{\text {mpcc }}$ if MPCC-GCQ for (E-MPCC) holds at $\left(t, w, u^{t}, v^{t}, u^{w}, v^{w}\right) \in \mathcal{F}_{\mathrm{e}-\mathrm{mpcc}}$ for any (and hence all) $\left(w, u^{w}, v^{w}\right) \in W\left(t, u^{t}, v^{t}\right)$.

Proof. The inclusion $\mathcal{T}_{\text {mpcc }}\left(t, u^{t}, v^{t}\right)^{*} \supseteq \mathcal{T}_{\text {mpcc }}^{\operatorname{lin}}\left(t, u^{t}, v^{t}\right)^{*}$ holds always. Thus, it is left to show that

$$
\mathcal{T}_{\text {mpcc }}\left(t, u^{t}, v^{t}\right)^{*} \subseteq \mathcal{T}_{\operatorname{mpcc}}^{\operatorname{lin}}\left(t, u^{t}, v^{t}\right)^{*}
$$

Let $\omega=\left(\omega t, \omega u^{t}, \omega v^{t}\right) \in \mathcal{T}_{\text {mpcc }}\left(t,, u^{t}, v^{t}\right)^{*}$, i.e. $\omega^{T} \delta \geq 0$ for all $\delta=\left(\delta t, \delta u^{t}, \delta v^{t}\right) \in \mathcal{T}_{\text {mpcc }}\left(t, u^{t}, v^{t}\right)$. Then, let $\tilde{\omega}=\left(\omega t, 0, \omega u^{t}, \omega v^{t}, 0,0\right)$ to obtain $\tilde{\omega}^{T} \tilde{\delta}=\omega^{T} \delta \geq 0$ for all $\tilde{\delta} \in \mathcal{T}_{\mathrm{e}-\operatorname{mpcc}}\left(t, w, u^{t}, v^{t}, u^{w}, v^{w}\right)$ where $w \in W(t)$ is arbitrary. By assumption, we have $\tilde{\omega}^{T} \tilde{\delta} \geq 0$ for all $\tilde{\delta} \in \mathcal{T}_{\mathrm{e}-\text { mpcc }}^{\operatorname{lin}}\left(t, w, u^{t}, v^{t}, u^{w}, v^{w}\right)$ which implies $\omega^{T} \delta=\tilde{\omega}^{T} \tilde{\delta} \geq 0$ for all $\delta \in \mathcal{T}_{\operatorname{mpcc}}^{\operatorname{lin}}\left(t, u^{t}, v^{t}\right)$.

The converse of the previous theorem is unlikely to hold, but we do not know how to construct a counterexample. However, equivalence of ACQ or GCQ for corresponding branch problems holds.

Theorem 3.14. ACQ for $\left(\operatorname{NLP}\left(\mathcal{P}^{t}\right)\right)$ holds at $\left(t, u^{t}, v^{t}\right) \in \mathcal{F}_{\mathcal{P} t}$ if and only if ACQ for $\left(\mathrm{NLP}\left(\mathcal{P}^{t, w}\right)\right)$ holds at $\left(t, w, u^{t}, v^{t}, u^{w}, v^{w}\right) \in \mathcal{F}_{\mathcal{P} t, w}$ for any (and hence all) $\left(w, u^{w}, v^{w}\right) \in W\left(t, u^{t}, v^{t}\right)$.

Proof. This follows immediately from Theorem 2.17, Theorem 4.3 and Theorem 4.7 .

Theorem 3.15. GCQ for $\left(\operatorname{NLP}\left(\mathcal{P}^{t}\right)\right)$ holds at $\left(t, u^{t}, v^{t}\right) \in \mathcal{F}_{\mathcal{P} t}$ if and only if GCQ for $\left(\operatorname{NLP}\left(\mathcal{P}^{t, w}\right)\right)$ holds at $\left(t, w, u^{t}, v^{t}, u^{w}, v^{w}\right) \in \mathcal{F}_{\mathcal{P} t, w}$ for any (and hence all) $\left(w, u^{w}, v^{w}\right) \in W\left(t, u^{t}, v^{t}\right)$.

Proof. This follows immediately from Theorem 2.18, Theorem 4.4 and Theorem 4.8.

\section{KINK QUALIFICATIONS AND MPCC CONSTRAINT QUALIFICATIONS}

In this section we show relations between abs-normal NLPs and counterpart MPCCs. Here, we consider both treatments of inequality constraints.

\subsection{RELATIONS OF GENERAL ABS-NORMAL NLP AND MPCC}

In the following the variables $x$ and $z$ instead of $t$ and $z^{t}$ are used. Thus, the abs-normal NLP (I-NLP) reads:

$$
\min _{x, z} f(x) \quad \text { s.t. } \quad c_{\mathcal{E}}(x,|z|)=0, \quad c_{\mathcal{I}}(x,|z|) \geq 0, \quad c_{\mathcal{Z}}(x,|z|)-z=0 .
$$

The counterpart MPCC (I-MPCC) becomes:

$$
\begin{array}{ll}
\min _{x, u, v} f(x) \quad \text { s.t. } \quad & c_{\mathcal{E}}(x, u+v)=0, \quad c_{\mathcal{I}}(x, u+v) \geq 0, \\
& c_{\mathcal{Z}}(x, u+v)-(u-v)=0, \quad 0 \leq u \perp v \geq 0 .
\end{array}
$$

Then, the subsequent relations of kink qualifications and MPCC constraint qualifications can be shown. 
Theorem 4.1 (AKQ for (I-NLP) $\Longleftrightarrow$ MPCC-ACQ for (I-MPCC)). AKQ for (I-NLP) holds at $(x, z(x)) \in$ $\mathcal{F}_{\text {abs }}$ if and only if MPCC-ACQ for (I-MPCC) holds at $(x, u, v)=\left(x,[z(x)]^{+},[z(x)]^{-}\right) \in \mathcal{F}_{\text {mpcc }}$.

Proof. We need to show

$$
\mathcal{T}_{\mathrm{abs}}(x, z)=\mathcal{T}_{\mathrm{abs}}^{\operatorname{lin}}(x, z) \Longleftrightarrow \mathcal{T}_{\text {mpcc }}(x, u, v)=\mathcal{T}_{\text {mpcc }}^{\operatorname{lin}}(x, u, v) .
$$

This is obvious from the homeomorphisms $\psi$ in Lemma 3.5.

Theorem 4.2 (MPCC-GCQ for (I-MPCC) implies GKQ for (I-NLP)). GKQ for (I-NLP) holds at $(x, z(x)) \in$ $\mathcal{F}_{\text {abs }}$ if MPCC-GCQ for (I-MPCC) holds at $(x, u, v)=\left(x,[z(x)]^{+},[z(x)]^{-}\right) \in \mathcal{F}_{\text {mpcc }}$.

Proof. The inclusion $\mathcal{T}_{\text {abs }}^{\operatorname{lin}}(x, z)^{*} \subseteq \mathcal{T}_{\text {abs }}(x, z)^{*}$ hold always by Lemma 2.8. Thus, we just have to show

$$
\mathcal{T}_{\text {abs }}(x, z)^{*} \subseteq \mathcal{T}_{\text {abs }}^{\operatorname{lin}}(x, z)^{*} .
$$

Consider $\omega=(\omega x, \omega z) \in \mathcal{T}_{\text {abs }}(x, z)^{*}$, i.e. $\omega^{T} \delta \geq 0$ for all $\delta=(\delta x, \delta z) \in \mathcal{T}_{\text {abs }}(x, z)$. Set $\tilde{\omega}=(\omega x, \omega z,-\omega z)$. For every $\delta \in \mathcal{T}_{\text {abs }}(x, z)$ we then have

$$
\tilde{\omega}^{T} \psi^{-1}(\delta)=\omega x^{T} \delta x+\omega z^{T}\langle\delta z\rangle^{+}-\omega z^{T}\langle\delta z\rangle^{-}=\omega x^{T} \delta x+\omega z^{T} \delta z=\omega^{T} \delta \geq 0 .
$$

This means $\tilde{\omega} \in \mathcal{T}_{\text {mpcc }}(x, u, v)^{*}$ and hence, by assumption, $\tilde{\omega} \in \mathcal{T}_{\text {mpcc }}^{\operatorname{lin}}(x, u, v)^{*}$. We thus have $\omega^{T} \delta=$ $\tilde{\omega}^{T} \psi^{-1}(\delta) \geq 0$ for every $\delta \in \mathcal{T}_{\text {abs }}^{\operatorname{lin}}(x, z)$, which means $\omega \in \mathcal{T}_{\text {abs }}^{\operatorname{lin}}(x, z)^{*}$.

The converse is unlikely to hold, although we are not, at this time, aware of a counterexample. Once again, moving to the branch problems allows to exploit additional sign information.

Theorem 4.3 (ACQ for $\left(\mathrm{NLP}\left(\Sigma^{t}\right)\right) \Longleftrightarrow \mathrm{ACQ}$ for $\left.\left(\operatorname{NLP}\left(\mathcal{P}^{t}\right)\right)\right)$. ACQ for $\left(\mathrm{NLP}\left(\Sigma^{t}\right)\right)$ holds at $(x, z(x)) \in \mathcal{F}_{\Sigma^{t}}$ if and only if $A C Q$ for the corresponding $\left(\operatorname{NLP}\left(\mathcal{P}^{t}\right)\right)$ holds at $(x, u, v)=\left(x,[z(x)]^{+},[z(x)]^{-}\right) \in \mathcal{F}_{\mathcal{P} t}$.

Proof. We need to show

$$
\mathcal{T}_{\Sigma^{t}}(x, z)=\mathcal{T}_{\Sigma^{t}}^{\operatorname{lin}}(x, z) \Longleftrightarrow \mathcal{T}_{\mathcal{P} t}(x, u, v)=\mathcal{T}_{\mathcal{P} t}^{\operatorname{lin}}(x, u, v) .
$$

This is obvious from the homeomorphisms $\psi \rho$ in Lemma 3.7.

Theorem 4.4 (GCQ for $\left(\mathrm{NLP}\left(\Sigma^{t}\right)\right) \Longleftrightarrow \mathrm{GCQ}$ for $\left.\left(\operatorname{NLP}\left(\mathcal{P}^{t}\right)\right)\right)$. GCQ for $\left(\mathrm{NLP}\left(\Sigma^{t}\right)\right)$ holds at $(x, z(x)) \in \mathcal{F}_{\Sigma^{t}}$ if and only if GCQ for the corresponding $\left(\operatorname{NLP}\left(\mathcal{P}^{t}\right)\right)$ holds at $(x, u, v)=\left(x,[z(x)]^{+},[z(x)]^{-}\right) \in \mathcal{F}_{\mathcal{P}}$.

Proof. The inclusions $\mathcal{T}_{\mathcal{P} t}^{\operatorname{lin}}(x, u, v)^{*} \subseteq \mathcal{T}_{\mathcal{P} t}(x, u, v)^{*}$ and $\mathcal{T}_{\Sigma^{t}}^{\operatorname{lin}}(x, z)^{*} \subseteq \mathcal{T}_{\Sigma^{t}}(x, z)^{*}$ hold always. Thus, we just have to show

$$
\mathcal{T}_{\Sigma^{t}}(x, z)^{*} \supseteq \mathcal{T}_{\Sigma^{t}}^{\operatorname{lin}}(x, z)^{*} \Longleftrightarrow \mathcal{T}_{\mathcal{P} t}(x, u, v)^{*} \supseteq \mathcal{T}_{\mathcal{P} t}^{\operatorname{lin}}(x, u, v)^{*} .
$$

First, we show the implication " $\Rightarrow$ ". Consider $\omega=(\omega x, \omega u, \omega v) \in \mathcal{T}_{\mathcal{P} t}(x, u, v)^{*}$, i.e. $\omega^{T} \delta \geq 0$ for all $\delta=(\delta x, \delta u, \delta v) \in \mathcal{T}_{\mathcal{P} t}(x, u, v)$. Set $\tilde{\omega}=(\omega x, \omega z)$ with

$$
\omega z_{i}= \begin{cases}+\omega u_{i}, & i \in \mathcal{U}_{+} \cup \mathcal{P} \\ -\omega v_{i}, & i \in \mathcal{V}_{+} \cup \overline{\mathcal{P}}\end{cases}
$$

This leads to

$$
\tilde{\omega}^{T} \psi_{\mathcal{P}}(\delta)=\omega x^{T} \delta x+\omega z^{T}(\delta u-\delta v)=\omega x^{T} \delta x+\omega u^{T} \delta u+\omega v^{T} \delta v=\omega^{T} \delta \geq 0
$$

for every $\delta \in \mathcal{T}_{\mathcal{P} t}(x, u, v)$, i.e. $\tilde{\omega} \in \mathcal{T}_{\Sigma^{t}}(x, z)^{*}$. Then, the assumption yields $\tilde{\omega} \in \mathcal{T}_{\Sigma^{t}}^{\operatorname{lin}}(x, z)^{*}$. As we have $\omega^{T} \delta=\tilde{\omega}^{T} \psi_{\mathcal{P}}(\delta) \geq 0$ for every $\delta \in \mathcal{T}_{\mathcal{P} t}^{\operatorname{lin}}(x, u, v)$, we obtain $\omega \in \mathcal{T}_{\mathcal{P} t}^{\operatorname{lin}}(x, u, v)^{*}$. The reverse implication follows as in Theorem 4.2. 


\subsection{RELATIONS OF ABS-NORMAL NLP AND MPCC WITH INEQUALITY SLACKS}

Now, the relations for the slack reformulations are stated. These are special cases of the general problem formulations, hence we obtain the following four theorems that correspond to Theorems 4.1 to 4.4. Theorem 4.5 (AKQ for (E-NLP) $\Longleftrightarrow$ MPCC-ACQ for (E-MPCC)). AKQ for (E-NLP) holds at $(x, z(x)) \in$ $\mathcal{F}_{\mathrm{e}-\mathrm{abs}}$ if and only if MPCC-ACQ for (E-MPCC) holds at $(x, u, v)=\left(x,[z(x)]^{+},[z(x)]^{-}\right) \in \mathcal{F}_{\mathrm{e} \text {-mpcc }}$.

Theorem 4.6 (MPCC-GCQ for (E-MPCC) implies GKQ for (E-NLP)). GKQ for (E-NLP) holds at $(x, z(x)) \in$ $\mathcal{F}_{\mathrm{e} \text {-abs }}$ if MPCC-GCQ for (E-MPCC) holds at $(x, u, v)=\left(x,[z(x)]^{+},[z(x)]^{-}\right) \in \mathcal{F}_{\mathrm{e}-\mathrm{mpcc}}$.

The converse is unlikely to hold, but to date we are not aware of a counterexample.

Theorem 4.7 (ACQ for $\left(\mathrm{NLP}\left(\Sigma^{t, w}\right)\right) \Longleftrightarrow \mathrm{ACQ}$ for $\left.\left(\mathrm{NLP}\left(\mathcal{P}^{t, w}\right)\right)\right)$. ACQ for $\left(\mathrm{NLP}\left(\Sigma^{t, w}\right)\right)$ at $(x, z(x)) \in$ $\mathcal{F}_{\Sigma^{t, w}}$ is equivalent to ACQ for the corresponding $\left(\operatorname{NLP}\left(\mathcal{P}^{t, w}\right)\right)$ at $(x, u, v)=\left(x,[z(x)]^{+},[z(x)]^{-}\right) \in \mathcal{F}_{\mathcal{P} t, w}$. Theorem $4.8\left(\mathrm{GCQ}\right.$ for $\left(\mathrm{NLP}\left(\Sigma^{t, w}\right)\right) \Longleftrightarrow \mathrm{GCQ}$ for $\left.\left(\mathrm{NLP}\left(\mathcal{P}^{t, w}\right)\right)\right)$. GCQ for $\left(\mathrm{NLP}\left(\Sigma^{t, w}\right)\right)$ at $(x, z(x)) \mathcal{F}_{\Sigma^{t, w}}$ is equivalent to GCQ for the corresponding $\left(\mathrm{NLP}\left(\mathcal{P}^{t, w}\right)\right)$ at $(x, u, v)=\left(x,[z(x)]^{+},[z(x)]^{-}\right) \in \mathcal{F}_{\mathcal{P} t, w}$.

All the relations discussed in Sections 2 to 4 are illustrated in Figure 1. In the inner square (containing (I-NLP) and (E-NLP) as well as the counterpart MPCCs (I-MPCC) and (E-MPCC)) there are four singleheaded arrows, which indicate that only one direction has been proved and we do not know if the converses hold as well. Therefore we considered the branch problems given on the outer right and left in the figure. Since ACQ respectively GCQ for all branch problems imply the corresponding kink qualification or MPCC-constraint qualification, there are further single-headed arrows pointing to the inner square. Results that follow directly from other equivalences have arrows with the label (implied).

\section{FIRST ORDER STATIONARITY CONCEPTS}

In this section, we introduce definitions of Mordukhovich stationarity and Bouligand stationarity for abs-normal NLPs and compare these definitions to M-stationarity and B-stationarity for MPCCs. We give proofs based on the general formulation.

\subsection{MORDUKHOVICH STATIONARITY}

In this paragraph we have a closer look at M-stationarity [13], which is a necessary optimality condition for MPCCs under MPCC-ACQ [2].

Definition 5.1 (M-Stationarity for (I-MPCC), see [13]). Consider a feasible point $\left(x^{*}, u^{*}, v^{*}\right)$ of (I-MPCC) with associated index sets $\mathcal{U}_{+}, \mathcal{V}_{+}$and $\mathcal{D}$. It is an M-stationary point if there exist multipliers $\lambda=$ $\left(\lambda_{\mathcal{E}}, \lambda_{\mathcal{I}}, \lambda_{\mathcal{Z}}\right)$ and $\mu=\left(\mu_{u}, \mu_{v}\right)$ such that the following conditions are satisfied:

$$
\begin{aligned}
\partial_{x, u, v} \mathcal{L}_{\perp}\left(x^{*}, u^{*}, v^{*}, \lambda, \mu\right) & =0, \\
\left(\left(\mu_{u}\right)_{i}>0,\left(\mu_{v}\right)_{i}>0\right) \vee\left(\mu_{u}\right)_{i}\left(\mu_{v}\right)_{i} & =0, i \in \mathcal{D} \\
\left(\mu_{u}\right)_{i} & =0, i \in \mathcal{U}_{+}, \\
\left(\mu_{v}\right)_{i} & =0, i \in \mathcal{V}_{+}, \\
\lambda_{I} & \geq 0, \\
\lambda_{I}^{T} c_{I}\left(x^{*}, u^{*}, v^{*}\right) & =0 .
\end{aligned}
$$

Herein, $\mathcal{L}_{\perp}$ is the MPCC-Lagrangian function

$$
\begin{aligned}
\mathcal{L}_{\perp}(x, u, v, \lambda, \mu):=f(x) & +\lambda_{\mathcal{E}}^{T} c_{\mathcal{E}}(x, u+v)-\lambda_{\mathcal{I}}^{T} c_{\mathcal{I}}(x, u+v) \\
& +\lambda_{\mathcal{Z}}^{T}\left[c_{\mathcal{Z}}(x, u+v)-(u-v)\right]-\mu_{u}^{T} u^{t}-\mu_{v}^{T} v^{t}
\end{aligned}
$$




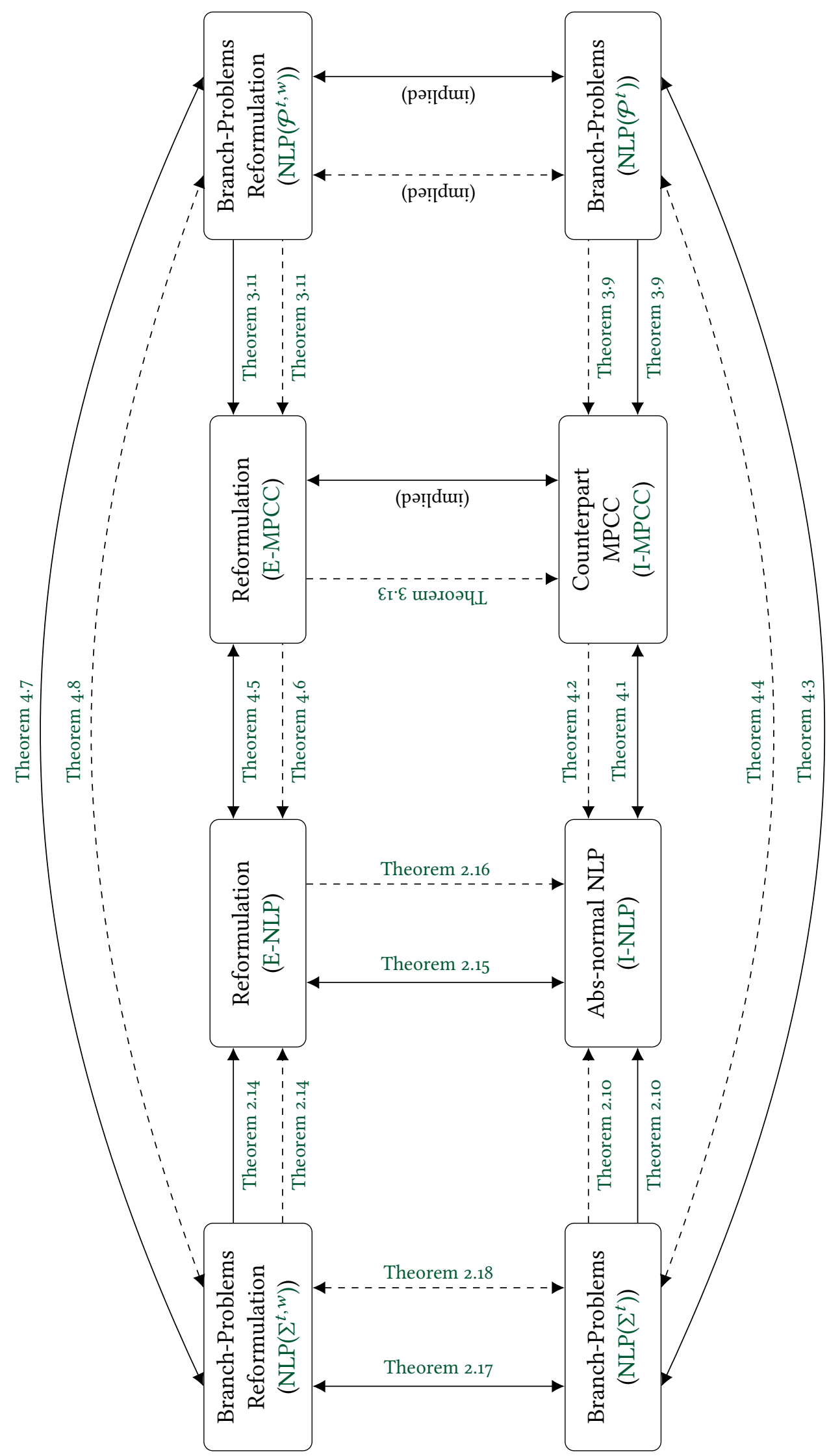

i.

है

हैํํ. 
Local minimizers of (I-MPCC) are M-stationary points under MPCC-ACQ, as shown in $[1,2]$. The name M-stationarity was introduced by Scholtes in [15] and was motivated by the fact that the sign restrictions on the multipliers in (5.1) in fact model the Mordukhovich normal cone. The inset on the right illustrates the feasible set of multiplier values for a pair $\left(\left(\mu_{u}\right)_{i},\left(\mu_{v}\right)_{i}\right), i \in \mathcal{D}$. M-stationarity is a weaker stationarity concept than strong stationarity, but is at the same time the strongest necessary optimality condition known to hold in absence of a strong constraint qualification like MPCC-MFCQ.

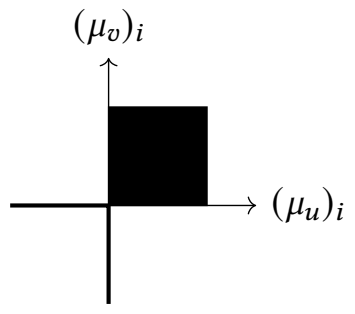

Definition 5.2 (M-Stationarity for (I-NLP)). Consider a feasible point $\left(x^{*}, z^{*}\right)$ of (I-NLP). It is an $M$ stationary point if there exist multipliers $\lambda=\left(\lambda_{\mathcal{E}}, \lambda_{I}, \lambda_{\mathcal{Z}}\right)$ such that the following conditions are satisfied:

$$
\begin{aligned}
f^{\prime}\left(x^{*}\right)+\lambda_{\mathcal{E}}^{T} \partial_{1} c_{\mathcal{E}}-\lambda_{I}^{T} \partial_{1} c_{I}+\lambda_{\mathcal{Z}}^{T} \partial_{1} c_{\mathcal{Z}} & =0, \\
{\left[\lambda_{\mathcal{E}}^{T} \partial_{2} c_{\mathcal{E}}-\lambda_{\mathcal{I}}^{T} \partial_{2} c_{I}+\lambda_{\mathcal{Z}}^{T} \partial_{2} c_{\mathcal{Z}}\right]_{i} } & =\left(\lambda_{\mathcal{Z}}\right)_{i} \sigma_{i}^{*}, i \notin \alpha\left(x^{*}\right), \\
\left(\mu_{i}^{-}\right)\left(\mu_{i}^{+}\right)=0 \vee\left[\lambda_{\mathcal{E}}^{T} \partial_{2} c_{\mathcal{E}}-\lambda_{I}^{T} \partial_{2} c_{I}+\lambda_{\mathcal{Z}}^{T} \partial_{2} c_{\mathcal{Z}}\right]_{i} & >\left|\left(\lambda_{\mathcal{Z}}\right)_{i}\right|, i \in \alpha\left(x^{*}\right), \\
\lambda_{I} & \geq 0, \\
\lambda_{I}^{T} c_{I} & =0 .
\end{aligned}
$$

Here we use the notation

$$
\begin{aligned}
& \mu_{i}^{+}:=\left[\lambda_{\mathcal{E}}^{T} \partial_{2} c_{\mathcal{E}}-\lambda_{\mathcal{I}}^{T} \partial_{2} c_{\mathcal{I}}+\lambda_{\mathcal{Z}}^{T}\left[\partial_{2} c_{\mathcal{Z}}-I\right]\right]_{i}, \\
& \mu_{i}^{-}:=\left[\lambda_{\mathcal{E}}^{T} \partial_{2} c_{\mathcal{E}}-\lambda_{\mathcal{I}}^{T} \partial_{2} c_{\mathcal{I}}+\lambda_{\mathcal{Z}}^{T}\left[\partial_{2} c_{\mathcal{Z}}+I\right]\right]_{i},
\end{aligned}
$$

and the constraints and the partial derivatives are evaluated at $\left(x^{*},\left|z^{*}\right|\right)$.

Theorem 5.3 (M-Stationarity for (I-MPCC) is M-Stationarity for (I-NLP)). A feasible point $\left(x^{*}, z^{*}\right)$ of (I-NLP) is M-stationary if and only if $\left(x^{*}, u^{*}, v^{*}\right)=\left(x^{*},\left[z^{*}\right]^{+},\left[z^{*}\right]^{-}\right)$of (I-MPCC) is M-stationary.

Proof. For indices that satisfy the first condition in (5.1b), the equivalence with the second condition in (5.2c) was shown in [9, Theorem 33]. Thus, we just need to consider the alternative conditions. For (I-MPCC) we have the relations

$$
\begin{aligned}
& {\left[\lambda_{\mathcal{E}}^{T} \partial_{2} c_{\mathcal{E}}-\lambda_{\mathcal{I}}^{T} \partial_{2} c_{\mathcal{I}}+\lambda_{\mathcal{Z}}^{T}\left[\partial_{2} c_{\mathcal{Z}}-I\right]\right]_{i}=\left(\mu_{u}\right)_{i}, i \in \mathcal{D},} \\
& {\left[\lambda_{\mathcal{E}}^{T} \partial_{2} c_{\mathcal{E}}-\lambda_{\mathcal{I}}^{T} \partial_{2} c_{\mathcal{I}}+\lambda_{\mathcal{Z}}^{T}\left[\partial_{2} c_{\mathcal{Z}}+I\right]\right]_{i}=\left(\mu_{v}\right)_{i}, i \in \mathcal{D},}
\end{aligned}
$$

which was also shown in [9, Theorem 33]. These are exactly the definitions of $\mu_{i}^{+}$and $\mu_{i}^{-}$in the definition of M-Stationarity for (I-NLP).

Consequently, we may now rephrase the result by $[1,2]$ in the language of abs-normal forms.

Theorem 5.4 (Minimizers and M-Stationarity for (I-NLP)). Assume that $\left(x^{*}, z^{*}\right)$ is a local minimizer of (I-NLP) and that AKQ holds at $x^{*}$. Then, $\left(x^{*}, z^{*}\right)$ is M-stationary for (I-NLP).

Proof. First, note that $\left(x^{*}, z^{*}\right)$ is a local minimizer of (I-NLP) if and only if $\left(x^{*}, u^{*}, v^{*}\right)=\left(x^{*},\left[z^{*}\right]^{+},\left[z^{*}\right]^{-}\right)$ is a local minimizer of (I-MPCC). Then, the point $\left(x^{*}, u^{*}, v^{*}\right)$ is a local minimizer of the counterpart MPCC, and MPCC-ACQ holds by Theorem 4.1. Thus, $\left(x^{*}, u^{*}, v^{*}\right)$ is M-stationary for (I-MPCC) and Theorem 5.3 implies that $\left(x^{*}, z^{*}\right)$ is M-stationary for (I-NLP). 


\subsection{MPCC-LINEARIZED BOULIGAND STATIONARITY}

Finally, we introduce MPCC-linearized Bouligand stationarity, which is defined via smooth subproblems. Definition 5.5 (MPCC-linearized B-Stationarity for (I-MPCC), see [14]). Consider a feasible point $\left(x^{*}, u^{*}, v^{*}\right)$ of (I-MPCC) with associated index sets $\mathcal{U}_{+}, \mathcal{V}_{+}$and $\mathcal{D}$. It is a $B$-stationary point if it is a stationary point of all branch problems $\left(\operatorname{NLP}\left(\mathcal{P}^{t}\right)\right)$ for $\mathcal{P}^{t}=\mathcal{P} \subseteq \mathcal{D}$. Here, $\overline{\mathcal{P}}$ denotes the complement of $\mathcal{P}$ in $\mathcal{D}\left(x^{*}\right)$.

Note that there exist different names for the variant of B-stationarity just introduced. It is simply called B-stationarity in [14], but we prefer here the name MPCC-linearized B-stationarity suggested in [1] to prevent confusion with the definition of B-stationarity in the smooth case. The concept of B-stationarity is the most intuitive among stationarity concepts in simply requiring that, no matter how degenerate pairs of complementarities are resolved, no first order descent direction may be revealed. Moreover, it may be brought into agreement with the concept of local minimizers already under very weak assumptions regarding constraint qualifications. The downside however is that verifying B-stationarity inherently requires exponential runtime effort, as the number of branch problems is exponential in the number of degenerate pairs in $\mathcal{D}$.

Theorem 5.6. If GCQ holds for all $\left(\mathrm{NLP}\left(\mathcal{P}^{t}\right)\right)$, then all local minimizers of (I-MPCC) are MPCC-linearized B-stationary points.

Proof. This follows directly by KKT theory for smooth optimization problems.

Definition 5.7 (Abs-Normal-Linearized B-Stationarity for (I-NLP)). Consider a feasible point $\left(x^{*}, z^{*}\right)$ of (I-NLP). It is an abs-normal-linearized B-stationary point if it is a stationary point of the branch problems $\left(\operatorname{NLP}\left(\Sigma^{t}\right)\right)$ for $\Sigma^{t}=\operatorname{diag}(\sigma)$ with $\sigma \geq \sigma(x)$.

Theorem 5.8 (MPCC-linearized B-stationarity for (I-MPCC) is abs-normal-linearized B-stationarity for (I-NLP)). A feasible point $\left(x^{*}, z^{*}\right)$ of (I-NLP) is abs-normal-linearized B-stationary if and only if $\left(x^{*}, u^{*}, v^{*}\right)=\left(x^{*},\left[z^{*}\right]^{+},\left[z^{*}\right]^{-}\right)$of (I-MPCC) is MPCC-linearized B-stationary.

Proof. Every branch problem $\left(\operatorname{NLP}\left(\Sigma^{t}\right)\right)$ is smooth and thus stationarity is equivalent to the condition $f^{\prime}\left(x^{*}\right)^{T} d \geq 0$ for all $d \in \mathcal{T}_{\Sigma^{t}}^{\operatorname{lin}}\left(x^{*}, z^{*}\right)$. Analogously, stationarity for every branch problem $\left(\operatorname{NLP}\left(\mathcal{P}^{t}\right)\right)$ is equivalent to the condition $f^{\prime}\left(x^{*}\right)^{T} d \geq 0$ for all $d \in \mathcal{T}_{\mathcal{P} t}^{\operatorname{lin}}\left(x^{*},\left[z^{*}\right]^{+},\left[z^{*}\right]^{-}\right)$. Then, the equivalence follows as both branch problems are homeomorphic and both linearization cones are homeomorphic by Lemma 3.7 .

Theorem 5.9 (Minimizers and abs-normal-linearized B-Stationarity for (I-NLP)). Assume that $\left(x^{*}, z^{*}\right)$ is a local minimizer of (I-NLP) and that GCQ holds at $\left(x^{*}, z^{*}\right)$ for all $\left(\mathrm{NLP}\left(\sum^{t}\right)\right)$. Then, $\left(x^{*}, z^{*}\right)$ is absnormal-linearized B-stationary for (I-NLP).

Proof. The point $\left(x^{*}, z^{*}\right)$ is a local minimizer of (I-NLP) if and only if $\left(x^{*}, u^{*}, v^{*}\right)=\left(x^{*},\left[z^{*}\right]^{+},\left[z^{*}\right]^{-}\right)$ is a local minimizer of (I-MPCC). Moreover, GCQ for all (NLP( $\left.\left.\Sigma^{t}\right)\right)$ and GCQ for all $\left(\operatorname{NLP}\left(\mathcal{P}^{t}\right)\right)$ are equivalent by Theorem 4.4 . Thus, $\left(x^{*}, u^{*}, v^{*}\right)$ is a local minimizer of the counterpart MPCC and GCQ holds for all $\left(\operatorname{NLP}\left(\mathcal{P}^{t}\right)\right)$. Then, it is MPCC-linearized B-stationary by Theorem 5.6 and finally $\left(x^{*}, z^{*}\right)$ is abs-normal-linearized B-stationary by Theorem 5.8 .

Remark 5.10. In [6], Griewank and Walther have presented a stationarity concept that holds without any kink qualification for minimizers of the unconstrained abs-normal NLP

$$
\min _{x} f(x), \quad f \in C_{\mathrm{abs}}^{d}\left(D^{x}, \mathbb{R}\right)
$$

Indeed, this concept is precisely abs-normal-linearized Bouligand stationarity: it requires the conditions of Definition 5.7 specialized to (5.3). Now, the question arises why no regularity assumption is needed. 
The answer is that the abs-normal form provides a certain degree of built-in regularity: we have shown in [8] that MPCC-ACQ is always satisfied for the counterpart MPCC of (5.3) (and thus every local minimizer is an M-stationary point). Analogously one can show that ACQ for all branch problems $\left(\operatorname{NLP}\left(\mathcal{P}^{t}\right)\right)$ is always satisfied for (5.3). Now, ACQ for all branch problems $\left(\mathrm{NLP}\left(\mathcal{P}^{t}\right)\right)$ is equivalent to ACQ for all branch problems (NLP( $\left.\left.\Sigma^{t}\right)\right)$ by Theorem $4 \cdot 3$, which in turn implies GCQ for all branch problems $\left(\operatorname{NLP}\left(\Sigma^{t}\right)\right)$. Thus, GCQ for all branch problems $\left(\operatorname{NLP}\left(\Sigma^{t}\right)\right)$ is always satisfied for $(5.3)$ and Theorem 5.9 holds.

\section{CONCLUSIONS}

We have shown that general abs-normal NLPs are essentially the same problem class as MPCCs. The two problem classes permit the definition of corresponding constraint qualifications, and optimality conditions of first order under weak constraint qualifications. We have also shown that the slack reformulation from [10] preserves constraint qualifications of Abadie type, whereas for Guginard type we could only prove some implications. Here, one subtle drawback is the non-uniqueness of slack variables. Thus, we have introduced branch formulations of general abs-normal NLPs and counterpart MPCCs. Then, constraint qualifications of Abadie and Guignard type are preserved.

\section{REFERENCES}

[1] M. Flegel, Constraint Qualifications and Stationarity Concepts for Mathematical Programs with Equilibrium Constraints, Dissertation, Universität Würzburg, 2005, https://opus.bibliothek.uni-wuerzburg.de/frontdoor/ index/index/year/2005/docld/1068.

[2] M. Flegel and C. Kanzow, A direct proof for M-stationarity under MPEC-ACQ for mathematical programs with equilibrium constraints, in Optimization with Multivalued Mappings, S. Dempe and V. Kalashnikov (eds.), volume 2 of Springer Optimization and Its Applications, Springer, Boston, MA, 2006.

[3] A. Griewank, On stable piecewise linearization and generalized algorithmic differentiation, Optimization Methods and Software 28 (2013), 1139-1178, doi:10.1080/10556788.2013.796683.

[4] A. Griewank and A. Walther, First- and second-order optimality conditions for piecewise smooth objective functions, Optimization Methods and Software 31 (2016), 904-930, doi:10.1080/10556788.2016.1189549.

[5] A. Griewank and A. Walther, Characterizing and testing subdifferential regularity for piecewise smooth objective functions, SIAM fournal on Optimization 29 (2019), 1473-1501, doi:10.1137/17m115520x.

[6] A. Griewank and A. Walther, Relaxing kink qualifications and proving convergence rates in piecewise smooth optimization, SIAM fournal on Optimization 29 (2019), 262-289, doi:10.1137/17m1157623.

[7] L. C. Hegerhorst-Schultchen, Optimality Conditions for Abs-Normal NLPs, Dissertation, Leibniz Universität Hannover, 2020, doi:10.15488/9867.

[8] L. C. Hegerhorst-Schultchen, C. Kirches, and M. C. Steinbach, On the relation between MPECs and optimization problems in abs-normal form, Optimization Methods and Software 35 (2020), 565-575, doi: 10.1080/10556788.2019.1588268.

[9] L. C. Hegerhorst-Schultchen, C. Kirches, and M. C. Steinbach, Relations between abs-normal NLPs and MPCCs. Part 1: Strong constraint qualifications, fournal of Nonsmooth Analysis and Optimization 2 (2021), 6672, doi:10.46298/jnsao-2021-6672.

[10] L. C. Hegerhorst-Schultchen and M.C. Steinbach, On first and second order optimality conditions for abs-normal NLP, Optimization 69 (2020), 2629-2656, doi:10.1080/02331934.2019.1626386. 
[11] J.Pang and M-Fukushima, Complementarity constraint qualifications and simplified B-stationarity conditions for mathematical programs with equilibrium constraints, Computational Optimization and Applications 13 (1999), 111-136, doi:10.1023/a:1008656806889.

[12] Z. Luo, J. Pang, and D. Ralph, Mathematical Programs with Equilibrium Constraints, Cambridge University Press, 1996.

[13] J. V. Outrata, Optimality conditions for a class of mathematical programs with equilibrium constraints, Mathematics of Operations Research 24 (1999), 627-644, doi:10.2307/3690651.

[14] H. Scheel and S. Scholtes, Mathematical programs with complementarity constraints: stationarity, optimality, and sensitivity, Mathematics of Operations Research 25 (2000), 1-22, doi:10.1287/moor.25.1.1.15213.

[15] S. Scholtes, Convergence properties of a regularization scheme for mathematical programs with complementarity constraints, SIAM fournal on Optimization 11 (2001), 918-936, doi:10.1137/s1052623499361233.

[16] J. Ye, Necessary and sufficient optimality conditions for mathematical programs with equilibrium constraints, fournal of Mathematical Analysis and Applications 307 (2005), 350-369, doi:10.1016/j.jmaa.2004.10. 032. 